ARTICLE

https://doi.org/10.1038/s41467-018-08222-6

\title{
Machine learning coarse grained models for water
}

\author{
Henry Chan (1) ${ }^{1}$, Mathew J. Cherukara (1) ${ }^{1}$, Badri Narayanan 1,3, Troy D. Loeffler ${ }^{1}$, Chris Benmore (1) ${ }^{2}$, \\ Stephen K. Gray ${ }^{1,4} \&$ Subramanian K.R.S. Sankaranarayanan ${ }^{1,4}$
}

An accurate and computationally efficient molecular level description of mesoscopic behavior of ice-water systems remains a major challenge. Here, we introduce a set of machine-learned coarse-grained (CG) models (ML-BOP, ML-BOP ${ }_{\text {dih, }}$ and $\mathrm{ML}-\mathrm{mW}$ ) that accurately describe the structure and thermodynamic anomalies of both water and ice at mesoscopic scales, all at two orders of magnitude cheaper computational cost than existing atomistic models. In a significant departure from conventional force-field fitting, we use a multilevel evolutionary strategy that trains CG models against not just energetics from first-principles and experiments but also temperature-dependent properties inferred from on-the-fly molecular dynamics ( 10's of milliseconds of overall trajectories). Our ML BOP models predict both the correct experimental melting point of ice and the temperature of maximum density of liquid water that remained elusive to-date. Our ML workflow navigates efficiently through the high-dimensional parameter space to even improve upon existing high-quality CG models (e.g. $\mathrm{mW}$ model).

\footnotetext{
${ }^{1}$ Center for Nanoscale Materials, Argonne National Laboratory, Argonne, IL 60439, USA. ${ }^{2}$ X-ray Science Division, Argonne National Laboratory, Argonne, IL 60439, USA. ${ }^{3}$ Department of Mechanical Engineering, University of Louisville, Louisville, KY 40292, USA. ${ }^{4}$ Consortium for Advanced Science and Engineering, University of Chicago, Chicago, IL 60637, USA. These authors contributed equally: Henry Chan, Mathew J. Cherukara, Badri Narayanan. Correspondence and requests for materials should be addressed to H.C. (email: hchan@anl.gov) or to S.K.R.S.S. (email: skrssank@anl.gov)
} 
ce nucleation and grain growth are ubiquitous phenomena. Ice nuclei, when formed, are nanoscopic ${ }^{1}$ - critical sizes start from tens of molecules-and subsequently consolidate into larger grains at the mesoscopic scale ${ }^{2}$. A molecular level picture of phase transformations in water, especially at mesoscopic scales, is most desirable but remains inaccessible to fully atomistic simulations ${ }^{3}$. The underlying phase transitions and dynamical processes in supercooled mesoscale systems are often inaccessible due to system size and timescale limitations, which are further compounded by their sluggish kinetics. While exascale computers may cope with such mesoscopic length scales, time scale challenges will remain (Supplementary Figure 1). It is important to have a water model that accurately captures the melting point, liquid and solid densities, as well as other thermodynamic and transport properties at modest computational cost. Numerous atomistic $^{4-6}$ and coarse-grained ${ }^{7}$ (CG) water models exist. They differ in terms of predictive power and computational cost/efficiency. The best performing non-polarizable atomistic model is TIP4P $/ 2005^{5}$. However, it under-predicts the melting point by $20 \mathrm{~K}$ and is too computationally expensive for large-scale molecular dynamics (MD) studies involving multi-million molecule ice-water systems. Polarizable models such as $\mathrm{MB}-\mathrm{pol}^{8}$ and AMOEBA $^{9}$ have comparable accuracy and can treat charged species, but are computationally expensive (Tables 1 and 2). CG models are computationally efficient, but often less accurate. The monoatomic water $(\mathrm{mW})$ model $^{10}$ remains the best performing CG model ${ }^{11}$, predicting the correct melting point and several thermodynamic properties, but does not quantitatively capture the density anomaly and over predicts density of ice (Supplementary Figure 2).

A correct description of water's complex properties with a potential model, especially in CG form, is challenging. Here, we introduce a machine-learning (ML) workflow (Fig. 1) that can be used to train models that accurately describe the behavior of ice and liquid water at mesoscopic scales. We develop a set of bondorder CG models (ML-BOP and ML-BOP $\mathrm{dih}_{\text {ih }}$ ) that are up to two orders of magnitude cheaper (Table 2, Supplementary Figure 3) than the most accurate non-polarizable atomistic models (TIP4P models and TIP5P) of comparable accuracy. As with the $\mathrm{mW}$ model ${ }^{10}$, our models treat each water molecule as one bead; the interactions between the beads are treated using a bond-order potential (BOP) both with and without explicit four-body term, i.e., on-the-fly dihedrals to describe tetrahedral solids. We use a multi-level hierarchical global optimization strategy to navigate the high-dimensional parameter space and train the ML models. We introduce ML models that adequately describe the thermodynamic and dynamical properties of water. Moreover, we also demonstrate that our ML strategy can be used to reoptimize existing high-quality water models, such as $\mathrm{mW}$, and improve their overall performance.

\section{Results}

Machine learning workflow for training CG water models. The ML workflow to train CG models involves three main aspects: Model selection, Training data generation, and Multi-level hierarchical objective optimization to parameterize models against target training data. The various stages involved in the ML workflow are discussed below.

CG model of water. Water molecules are modeled using a 1:1 CG model. The mapping of atomistic water molecules into CG water beads is done via the removal of hydrogen atoms, such that the CG beads are positioned at the positions of the oxygen atoms. This representation of water molecules as monoatomic beads and the use of the ML models can lead to a much more significant speed-up in MD simulations than the naive factor of three from

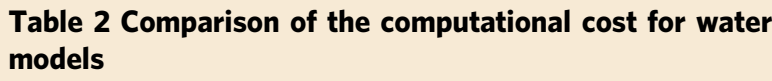

The benchmark system is liquid water ( 256 molecules) at $298 \mathrm{~K}$

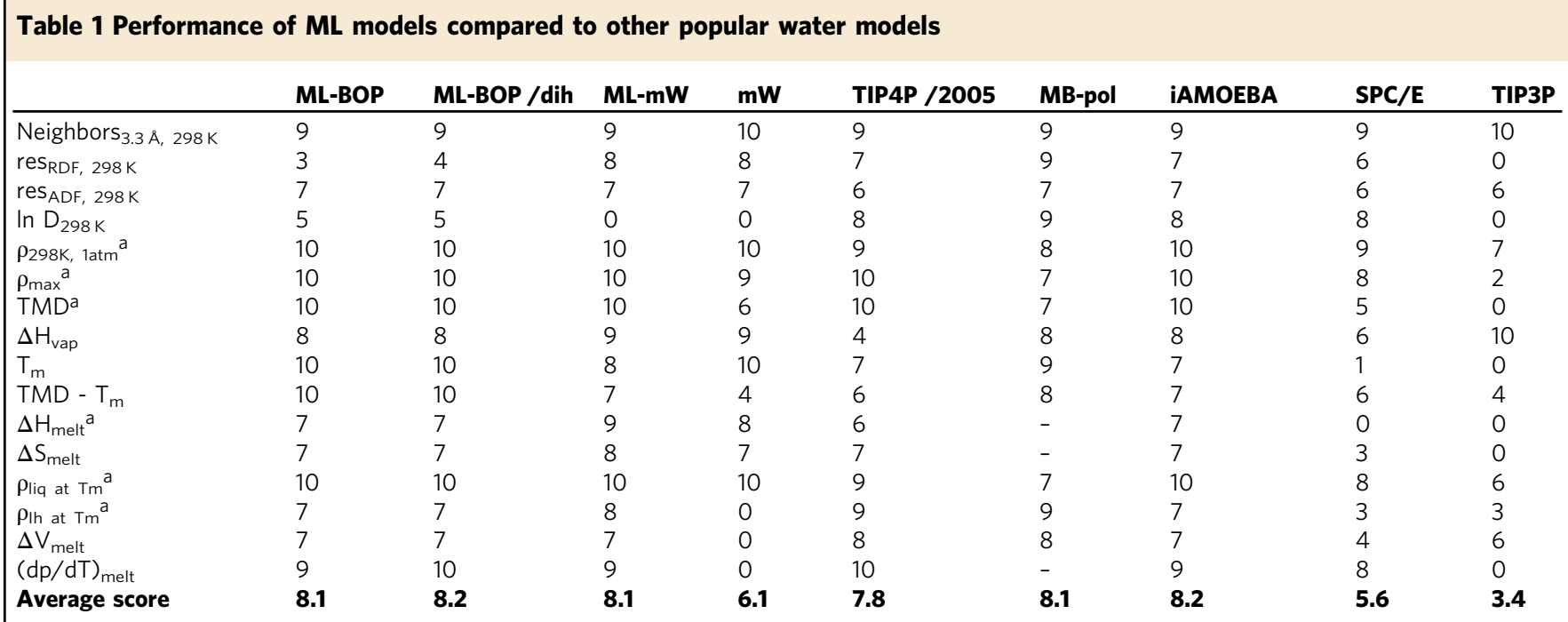

Comparison of the performance of ML models with other popular polarizable ${ }^{8,19}$ and non-polarizable models ${ }^{26}$. The numerical scores and tolerance are assigned based on an established system by $\mathrm{Vega}^{59}$. A list of ice and liquid water properties relevant to the capability of ML-BOP models are selected for comparison

aproperties that are included in the training of ML models 
a

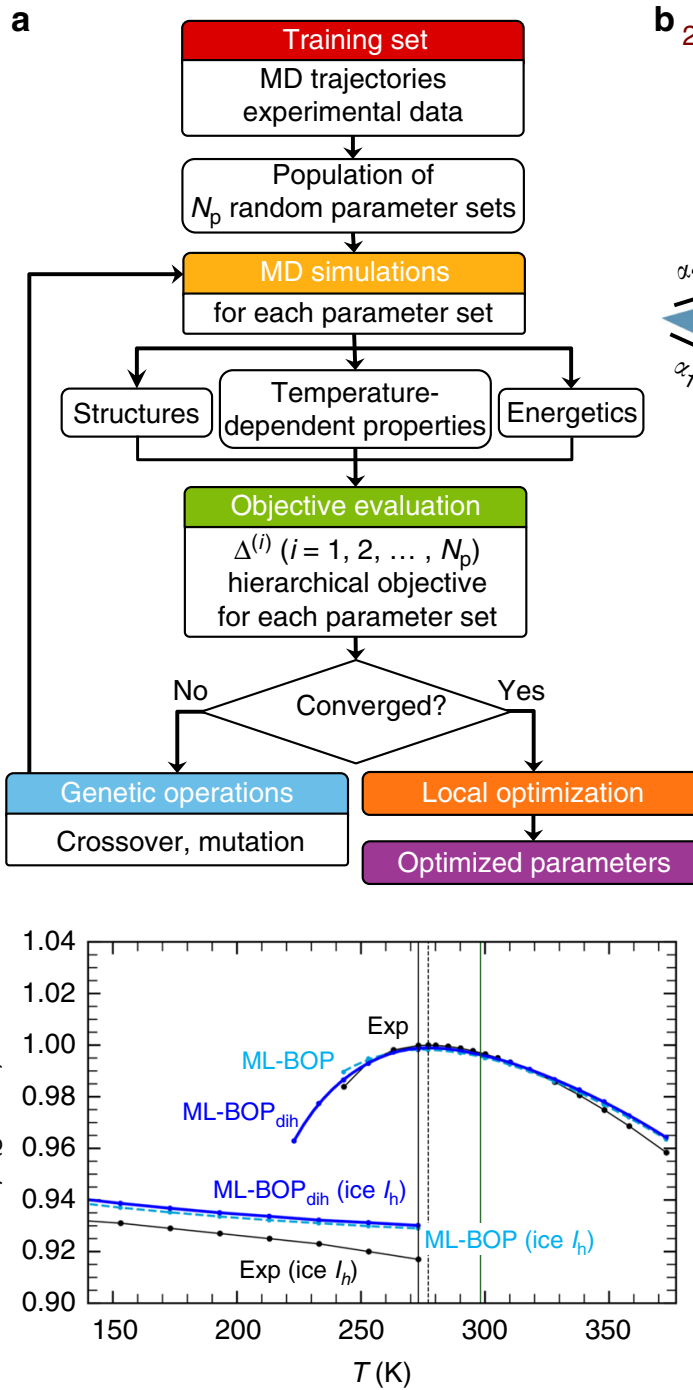

2-stage optimization technique

\section{Global optimization (GA)}

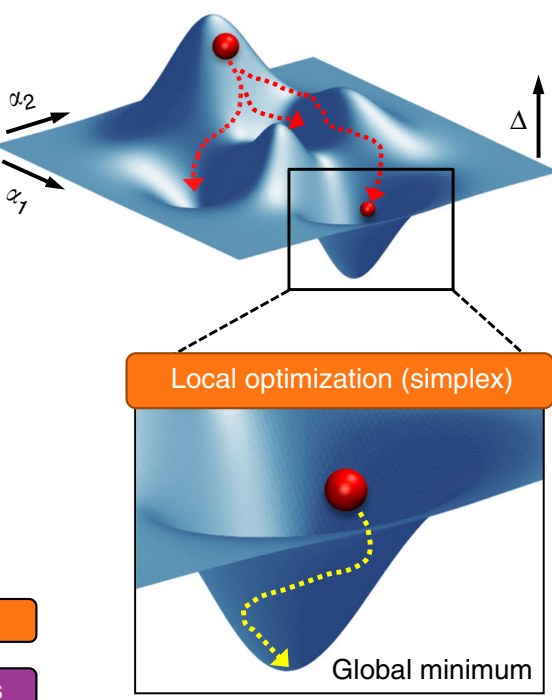

\section{e}
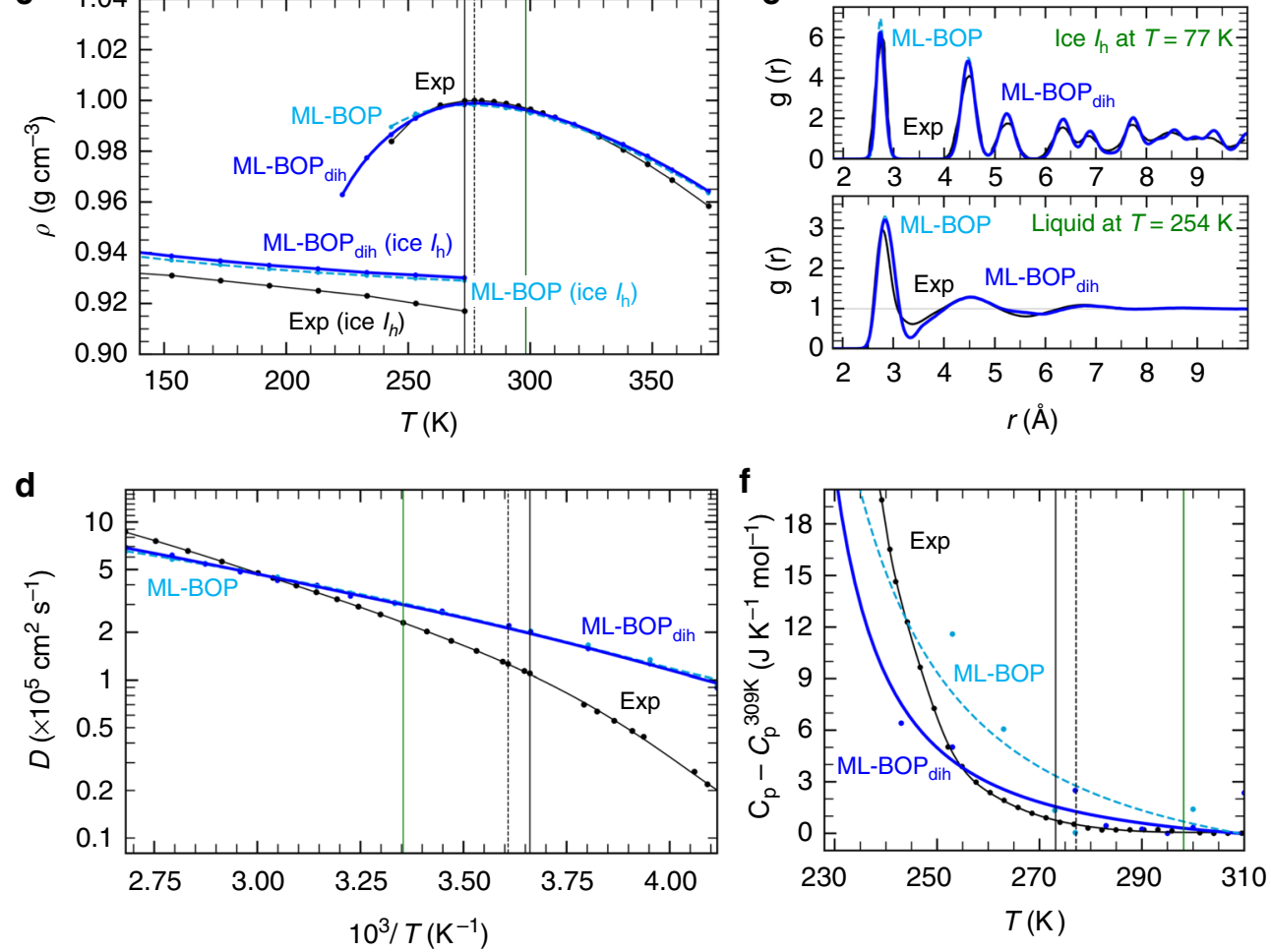

Fig. 1 Machine learning protocol to train water potentials and comparison with experiments. a Workflow depicting force field parameterization. One novelty is a direct fitting to dynamically-inferred properties through long time scale MD simulations. $N_{p}$ refers to population size and $\Delta^{(i)}$ refers to errors computed for the $i^{\text {th }}$ parameter set in the $N_{p}$ population using hierarchical objective. b Diagrams illustrating the 2-stage technique for locating the global minimum of the objective landscape. (The actual optimization involves up to 17 parameters but here we indicate just two generic parameters, $\alpha_{1}$ and $\alpha_{2}$.) Table 3 has the optimized ML-BOP and ML-BOP dih parameters. In (c-f) the experimental (Exp) melting point $(T=273 \mathrm{~K})$, maximum density temperature $(T=277 \mathrm{~K})$, and room temperature $(T=298 \mathrm{~K})$ are vertical solid black, dotted black, and solid green lines, respectively. c ML-BOP models accurately reproduce the density anomaly of water within $1.4 \%$ as shown by comparison with experimental densities ${ }^{55}$ of ice and liquid water at pressure 1 bar. Melting point of ML-BOP models is $273 \pm 1 \mathrm{~K}$. d ML-BOP models predict the experimental diffusion coefficients of water 20,56 over a wide temperature range. e ML-BOP models reproduce the experimental radial distribution functions of ice at $T=77 \mathrm{~K}^{57}$ and liquid water at $T=254 \mathrm{~K}^{23}$. $\mathbf{f} \mathrm{ML-BOP}$ models capture the experimental heat capacity of water $^{58}$ relative to the value at $T=309 \mathrm{~K}$ 
the spatially reduced number of atoms. This is a result of larger simulation time steps being possible due to the absence of fast $\mathrm{O}-\mathrm{H}$ vibrations, a significantly reduced number of pairwise interactions due to the reduced number of atoms, and the very simple CG potential form. A 1:1 CG model of water achieves both simplicity and computational efficiency.

Machine learned bond-order potential for CG water. The MLBOP model is based on the Tersoff-Brenner formalism ${ }^{12}$ (Pauling bond-order concept), which is used here to describe the shortrange directional interactions between CG water beads. The pair potential function $V_{\text {pair }}$ is given by

$$
V_{\text {pair }}=\mathrm{f}_{\mathrm{C}}\left(r_{i j}\right)\left[\mathrm{f}_{\mathrm{R}}\left(r_{i j}\right)+\mathrm{b}_{i j} \mathrm{f}_{\mathrm{A}}\left(r_{i j}\right)\right]
$$

where $\mathrm{f}_{\mathrm{C}}\left(r_{i j}\right), \mathrm{f}_{\mathrm{R}}\left(r_{i j}\right)$, and $\mathrm{f}_{\mathrm{A}}\left(r_{i j}\right)$ are the cutoff, repulsive, and attractive pair interactions, respectively, between bead $i$ and $j$ separated by a distance $r_{i j}$, and $b_{i j}$ is a bond-order parameter which modifies the pair interaction strength between bead $i$ and $j$ depending on their local chemical environment.

The cutoff function limits the range of interaction mainly to improve computational efficiency. The function is given by

$$
\mathrm{f}_{\mathrm{C}}(r)=\left\{\begin{array}{cc}
1, & r<R-D \\
\frac{1}{2}-\frac{1}{2} \sin \left(\frac{\pi(r-R)}{2 D}\right), & R-D<r<R+D \\
0, & r>R+D
\end{array}\right.
$$

where $R$ and $D$ are free parameters that are chosen to include only the first nearest neighbors, such that their pair interactions are smoothly reduced starting from the distance $R-D$ and are completely turned off beyond the distance $R+D$.

The repulsive and attractive pair interactions between CG water beads are modeled using exponential decay functions given by

$$
\begin{gathered}
\mathrm{f}_{\mathrm{R}}(r)=A \mathrm{e}^{-\lambda_{1} r} \\
\mathrm{f}_{\mathrm{A}}(r)=-B \mathrm{e}^{-\lambda_{2} r}
\end{gathered}
$$

where $A, B, \lambda_{1}$, and $\lambda_{2}$ are free parameters that control the overall strength and length scale of the repulsive and attractive potentials. Furthermore, the strength of $\mathrm{f}_{\mathrm{A}}(r)$ between beads $i$ and $j$ is scaled by a bond-order term $\mathrm{b}_{i j}$ which is given by

$$
\begin{gathered}
\mathrm{b}_{i j}=\left(1+\beta^{n} \xi_{i j}^{n}\right)^{-\frac{1}{2 n}} \\
\xi_{i j}=\sum_{k \neq i, j} \mathrm{f}_{\mathrm{C}}\left(r_{i k}\right) \mathrm{g}\left(\theta_{i j k}\right) \\
\mathrm{g}(\theta)=1+\frac{c^{2}}{d^{2}}-\frac{c^{2}}{\left[d^{2}+\left(\cos \theta-\cos \theta_{0}\right)^{2}\right]}
\end{gathered}
$$

where $\beta, n, c, d$, and $\cos \theta_{0}$ are free parameters. $\xi_{i j}$ defines the effective coordination of bead $i$, taking into account the relative distances $r_{i k}$ and interatomic angles $\theta_{i j k}$ of its neighboring beads. The three-body angular dependence is described by the function $g$ $(\theta)$, which has minima defined by $\cos \theta_{0}$ and the strength and sharpness of its effect controlled by $c$ and $d$.

Machine learned bond-order potential with on-the-fly dihedrals. Typical potentials (e.g., Stillinger-Weber or bond-order based such as Tersoff as described above) are based on first nearest neighbor interactions and hence the functional forms do not explicitly distinguish (energetically) between cubic and hexagonal ice structures. To address this limitation, we extend the
Tersoff bond-order potentials to include on-the-fly dihedral calculations similar to that implemented in AIREBO type models ${ }^{13}$. The dihedral potential function is described by

$$
V_{\text {dihedral }}(\varphi)=k_{\operatorname{dih}}\left[\sin ^{3 p}\left(\frac{\varphi}{2}\right)-\cos ^{p} \varphi\right]
$$

where $k_{\text {dih }}$ is the minimum well-depth, $p$ controls the steepness of the well, and $\varphi$ is the dihedral angle. In contrast to the dihedral potential functions typically used in rigid-bond models, the welldepth (and number of minima) of this potential changes depending on the number and local coordination of water beads. To improve computational efficiency and to handle any discontinuities due to this reactive characteristic, the Tersoff cutoff function, $\mathrm{f}_{\mathrm{C}}(r)$, is applied to every pair of water beads constituting a dihedral angle and an angular cutoff function, $f_{D}(\theta)$, is applied to every triplet of those water beads.

$$
\begin{gathered}
\mathrm{f}_{\mathrm{D}}(\theta)=\left\{\begin{array}{cr}
1, & \cos \theta_{2 \mathrm{a}} \leq \cos \theta \leq \cos \theta_{1 \mathrm{~b}} \\
\mathrm{t}_{1}^{2}\left(3-2 \mathrm{t}_{1}\right), & \cos \theta_{1 \mathrm{~b}}<\cos \theta<\cos \theta_{1 \mathrm{a}} \\
1-\mathrm{t}_{2}^{2}\left(3-2 \mathrm{t}_{2}\right), & \cos \theta_{2 \mathrm{~b}}<\cos \theta<\cos \theta_{2 \mathrm{a}} \\
0, & \cos \theta_{1 \mathrm{a}} \leq \cos \theta \leq \cos \theta_{2 \mathrm{~b}}
\end{array}\right. \\
\mathrm{t}_{1}=\frac{\cos \theta-\cos \theta_{1 \mathrm{a}}}{\cos \theta_{1 \mathrm{~b}}-\cos \theta_{1 \mathrm{a}}}, \mathrm{t}_{2}=\frac{\cos \theta-\cos \theta_{2 \mathrm{a}}}{\cos \theta_{2 \mathrm{~b}}-\cos \theta_{2 \mathrm{a}}}
\end{gathered}
$$

where $\cos \theta_{1 \mathrm{a}}$ and $\cos \theta_{2 \mathrm{~b}}$ define the lower and upper bounds of the angular cutoff analogous to $R+D$ in $\mathrm{f}_{\mathrm{C}}(r), \cos \theta_{1 \mathrm{~b}}$ and $\cos \theta_{2 \mathrm{a}}$ define the switching angle for the lower and upper bound angular cutoffs analogous to $R-D$ in $\mathrm{f}_{\mathrm{C}}(r)$.

Model parameterization. The parameterization of ML-BOP for water requires simultaneous optimization of 11 free parameters $\left(R, D, A, B, \lambda_{1}, \lambda_{2}, \beta, n, c, d, \cos \theta_{0}\right)$. Likewise, the parameterization of ML-BOP ${ }_{\text {dih }}$ for water requires optimization of 17 free parameters $\left(R, D, A, B, \lambda_{1}, \lambda_{2}, \beta, n, c, d, \cos \theta_{0}, \cos \theta_{1 \mathrm{a}}, \cos \theta_{1 \mathrm{~b}}, \cos \theta_{2 \mathrm{a}}\right.$, $\left.\cos \theta_{2 \mathrm{~b}}, k, p\right)$, which makes independent fitting of the parameters infeasible. Most of these parameters do not correspond to physical properties of the system, so they cannot be chosen based on intuition. In this work, we employ global and local optimization techniques and state-of-the-art machine learning principles to search for an optimized parameter set for water as described below.

Multi-level hierarchical objective machine learning workflow. Our machine learning workflow to train the CG models is illustrated in Fig. 1. In our training scheme (Fig. 1a), we introduce a multilevel evolutionary strategy (hierarchical objective genetic algorithm-HOGA) to train the ML models against an extensive training data set of energies and structural properties of ice and liquid water derived from the best available atomistic model (TIP4P/2005), supplemented by experimental data. The training data in the case of $\mathrm{ML}-\mathrm{BOP}_{\mathrm{dih}}$ also includes first principles energetic differences reported for cubic and hexagonal ice phases ${ }^{14}$. This elaborate training data set ensures an adequate representation of the diverse configurational space of ice and liquid water while amply sampling the energy landscape. We use HOGA to perform a global search followed by local optimization to find the optimized model parameters (Fig. 1b). This circumvents problems encountered with the local minimizers often used in force field fitting that rely on good starting guesses. An important new aspect of our scheme is that the iterations involve not just static evaluations of potential properties but also temperature-dependent properties sampled dynamically from several MD trajectories during the evolutionary process (10's of 
milliseconds of overall MD trajectories). HOGA aids in an accelerated evolutionary search by efficiently sampling the parameter landscape within a given GA generation, and overcoming the limitation of assigning arbitrary weights within a single objective thereby ensuring that all the properties (static or dynamic) are equally well described.

Training data set. The machine learning workflow begins with the preparation of an extensive data set, which is necessary for a supervised training method. We build the training set from atomistic MD trajectories of 1600 TIP4P/2005 water molecules, simulated at pressure $P=1$ bar over a wide range of temperatures using the LAMMPS simulator ${ }^{15}$ with a $13 \AA$ interaction cutoff, the particle-particle particle-mesh method for long-range electrostatic interactions, and a $1 \mathrm{fs}$ time step. The training set consists of various ice and liquid water configurations, which includes hexagonal ice at $123 \mathrm{~K}<T<273 \mathrm{~K}$, supercooled liquid water at $253 \mathrm{~K}<T<273 \mathrm{~K}$, normal condensed phase liquid water at $273 \mathrm{~K}<T<373 \mathrm{~K}$, and ice-water interfaces. Unlike most typical force field fitting procedures, we fit to the structure and energetics of TIP4P/2005 water configurations in the training set but also go beyond that by using the TIP4P/2005 training set as good starting configurations for running MD simulations with ML models during the fitting process. Properties including dynamical properties can be sampled from these simulations and be used to fit directly to experimental values of thermodynamic properties. Note that the main limitation of the TIP4P/2005 model is its inability to get the correct melting point $\left(T_{\mathrm{m}}\right)$ and the relative difference between temperature of maximum density (TMD) and $T_{\mathrm{m}}$. In such cases, we use known experimental values as targets.

All MD simulations performed during our force field fitting workflow are run in an isobaric-isothermal ensemble at pressure $P=1$ bar and different target temperatures using the LAMMPS simulator ${ }^{15}$. The equilibration time of these simulations varies from $150 \mathrm{ps}$ to $4 \mathrm{~ns}$ depending on the configuration and temperature (e.g., shortest for ice and longest for supercooled water). Note that the training set contains configurations of ice and liquid water over a wide range of temperatures, static properties as well as time-averaged properties such as $\Delta \mathrm{H}_{\mathrm{m}}$ and $\rho_{\mathrm{i}}$ sampled from MD simulations.

Hierarchical objective genetic algorithm (HOGA). The quality of a proposed parameter set is evaluated based on a hierarchical objective function (see pseudo code in Supplementary Note 1). In the HOGA evolutionary scheme, we truncate the evaluation of a parameter set which leads to large errors in hierarchical property classes and assign it a penalty depending on which class it fails at. The selection of hierarchical classes is at the discretion of the user. In this case, the hierarchy of the property classes is as listed in Supplementary Table 2. Note that a higher preference is given to the temperature-dependent densities of ice, water and the melting point to ensure that the models reproduce the density anomaly and the relative locations of melting point and TMD. The hierarchical approach aids in an accelerated evolutionary search by efficiently sampling the parameter landscape within a given generation, and overcoming the limitation of assigning arbitrary weights within a single objective thereby ensuring that all the properties (static or dynamic) are equally well described.

Given the objective function definition as described above, we proceed to apply a two-stage optimization technique to search for a suitable parameter set for water in the multi-dimensional parameter space. The goal is to locate the global minimum in the objective value landscape. We strategically start with a broad survey of the landscape using global optimization methods followed by a deeper refinement search using local optimization methods. In principle, any combination of global and local optimization methods should work for such a workflow. Here, we choose to use the genetic algorithm ${ }^{16}$ (GA) for global optimization and the Nelder-Mead simplex algorithm ${ }^{17}$ for local optimization.

Using HOGA, the global optimization process begins with the initialization of a population of $N_{\mathrm{p}}$ random parameter sets. The objective value $\Delta^{(\mathrm{i})}$ for each of these parameter sets is evaluated and their convergence is checked. If the convergence criteria are not met, then a new list of $N_{\mathrm{p}}$ parameter sets is derived using genetic operations (selection, cross-over, mutation, etc.) from the $\mathrm{m}$ old parameter sets having the lowest objective values. The selection operation creates a list of best parameter sets based on their objective values, which mimics the principle of "survival of the fittest" in evolution. The crossover operation intermixes these parameter sets to generate new potential good candidates, analogous to how good traits are passed from biological parents to their offspring. The mutation operation introduces sufficient diversity into the population to avoid pre-mature convergence of the GA run, which also provides the population the opportunity to improve beyond those possible via inheriting traits from parent structures (crossover). In this work, we used tournament selection without replacement as the selection operation, the simulated binary method as the crossover operation with an operation probability of 0.9 , and a polynomial of order 20 for the mutation operation with an operation probability of 0.1 . The objective value is evaluated for the new parameter sets followed by convergence test. This routine is iteratively performed until convergence.

To effectively survey the objective landscape, we typically perform at least $20 \mathrm{GA}$ runs simultaneously (up to a total of 100 runs), where each GA run has a population size of 200 and run for about 100 generations. The global optimization stage typically returns a list of close-to-optimal parameter sets which we further refine using local optimization techniques. In this work, we use the Nelder-Mead simplex algorithm ${ }^{17}$ for local optimization, and the final parameter set is chosen based on the performance in validation tests. The best parameter sets for ML-BOP and ML$\mathrm{BOP}_{\text {dih }}$ optimized through HOGA are provided in Table 3.

Model validation and performance of machine learned CG water models. Figure 1c, d compares structural and dynamicsinferred properties with experimental data. Our ML-BOP models successfully capture the best-known thermodynamic anomaly, the existence of a density maximum at $277 \mathrm{~K}$ (Fig. 1c); they correctly describe the freezing/melting transition at $273 \pm 1 \mathrm{~K}$, and densities of ice $(140 \mathrm{~K}-273 \mathrm{~K})$ and water $(243 \mathrm{~K}-373 \mathrm{~K})$ within $1.4 \%$ of experiments. Capturing the correct value of the TMD relative to the melting point has remained a challenge for all water models $10,18,19$. TIP4P/2005 is the best atomistic model to depict TMD but underestimates the melting point by $20 \mathrm{~K}$. Regarding transport properties (Fig. 1d), the room temperature diffusivity, ML-BOP models is $\sim 3 \times 10^{-5} \mathrm{~cm}^{2} \mathrm{~s}^{-1}$ in close agreement with experiment ${ }^{20}\left(2.3 \times 10^{-5} \mathrm{~cm}^{2} \mathrm{~s}^{-1}\right)$. Both ML models slightly overestimate diffusivities in the supercooled range but outperform other existing water models (Supplementary Figure $2 b$ ).

Figure le compares the $\mathrm{O}-\mathrm{O}$ radial distribution function (RDF) for ice $\mathrm{I}_{\mathrm{h}}$ at $77 \mathrm{~K}$ and (supercooled) liquid water at $254 \mathrm{~K}$ derived from experiments. The location and intensities of the peaks corresponding to first, second and third coordination shells are in good agreement. ML-BOP models, however, over-structure water, and underestimate the exchange of water molecules between first and second coordination shell ${ }^{21,22}$ (deeper minimum in the radial distribution function or RDF at $\sim 3.4 \AA$ ). Our 
Table 3 Force field parameters of ML models optimized using our developed workflow

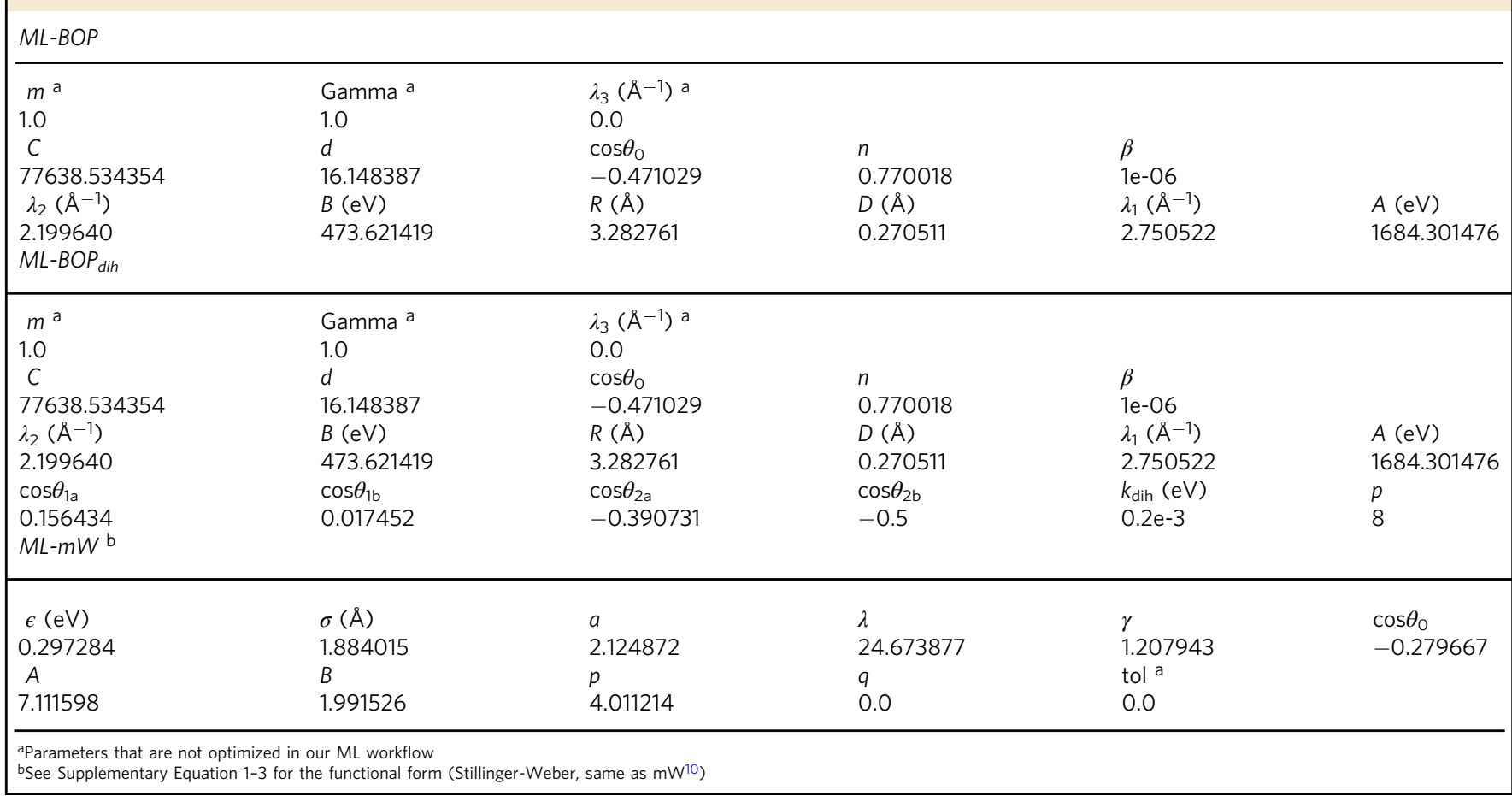

model is suitable for mesoscopic phenomena, such as ice nucleation and grain growth as well as applications involving polycrystalline ice, e.g., friction, mechanics of ice, melting of ice crystals, or pollutant effects on nucleation and ice grain growth (for example, see Supplementary Figure 4). The model captures the temperature and pressure dependent (Supplementary Figure 6) trends of these peaks. The ML-BOP calculated number of water neighbors in the first solvation shell, integrated out to the predicted temperature independent isosbestic point $(r=3.25 \AA$ ), is 4.7 in accordance with the experimental range of $4.3-4.7^{23,24}$. Also, the angular distribution function at $298 \mathrm{~K}$ agrees well with TIP4P/2005 (Supplementary Figure 5b). The ML-BOP heat capacities for liquid water, with respect to their values at $309 \mathrm{~K}$, reproduce the thermodynamic anomaly indicated by the sharp increase in $C_{p}$ of supercooled water (Fig. 1f). We also introduce an ML-BOP $_{\text {dih }}$ which represents a modification of ML-BOP model to include on-the-fly dihedrals. $\mathrm{ML}-\mathrm{BOP}_{\mathrm{dih}}$ performs on par with ML-BOP and additionally was trained using HOGA to capture the DFT predicted free energy difference $(\sim 1.4 \mathrm{meV} /$ atom per water molecule) between ice polymorphs. The performances of both ML-BOP and ML-BOP ${ }_{\text {dih }}$ are detailed in Tables 4-6 and Supplementary Figure 2-6. Overall, the trained ML models perform better or on par with the best available water models in several of the properties listed, but at a fraction of the computational cost.

HOGA to retrain existing best performing CG models. Our machine learning strategy is quite general and can be used to improve a variety of existing material models. To demonstrate this capability, we retrain the best available coarse grained model, for water, i.e. the $\mathrm{mW}$ model ${ }^{10}$, against our training data-set using the HOGA ML workflow. The new mW model trained using the machine learning workflow (termed ML-mW and given in Table 3 ) correctly captures the TMD, the density and structure of ice in the supercooled regime $(140-270 \mathrm{~K})$ as well as improves several thermodynamic and transport properties compared to

\begin{tabular}{|c|c|}
\hline & $\gamma\left(\mathrm{m} J \mathrm{~m}^{-2}\right)$ \\
\hline Exp & $29-33^{a}$ \\
\hline ML-BOP & 26.3 \\
\hline$M L-B O P_{d i h}$ & 26.8 \\
\hline $\mathrm{ML}-\mathrm{mW}$ & 29.3 \\
\hline $\mathrm{mW}$ & $35^{\mathrm{b}}$ \\
\hline TIP4P/2005 & $29^{b}$ \\
\hline TIP4P/Ice & $30^{b}$ \\
\hline TIP4P-Ew & $37 c$ \\
\hline TIP5P & $42^{c}$ \\
\hline
\end{tabular}

original $\mathrm{mW}$ while retaining the structure (e.g., RDF) of liquid $\mathrm{mW}$ water. The limitation of the ML-mW is that the melting point is slightly over-predicted $(\sim 289 \mathrm{~K})$ and, in contrast with the

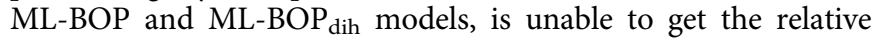
difference between $T_{\mathrm{m}}$ and TMD. Nevertheless, the overall predictions of ML-mW are better than the original $\mathrm{mW}$ in several properties (see Fig. 2 and Tables 1 and 5).

Briefly, the ML trained $\mathrm{mW}$ improves upon several of the properties compared to the original $\mathrm{mW}$. For example, the diffusion coefficient at $300 \mathrm{~K}$ is $4.8 \times 10^{-5} \mathrm{~cm}^{2} \mathrm{~s}^{-1}$, which is closer to the experimental value of $2.4 \times 10^{-5} \mathrm{~cm}^{2} \mathrm{~s}^{-1}$. Likewise, the density of ice at the melting point improves from 0.978 to $0.930 \mathrm{~g} \mathrm{~cm}^{-3}$, which is closer to the experimental ice density $\left(0.917 \mathrm{~g} \mathrm{~cm}^{-3}\right)$. The volume change upon melting, the TMD as well as the enthalpy of melting show an improvement over the original $\mathrm{mW}$ model. Other properties such as $(\mathrm{dp} / \mathrm{dT})_{\text {melt }}$ also show a significant improvement as detailed in Table 5 . Note that these improved predictions come by sacrificing the melting point; the ML-mW predicts the melting point to be $289 \mathrm{~K}$ which is $16 \mathrm{~K}$ higher than the original $\mathrm{mW}$ and experimental melting point. 
Table 5 Properties of ML models compared to experiments and other popular water models

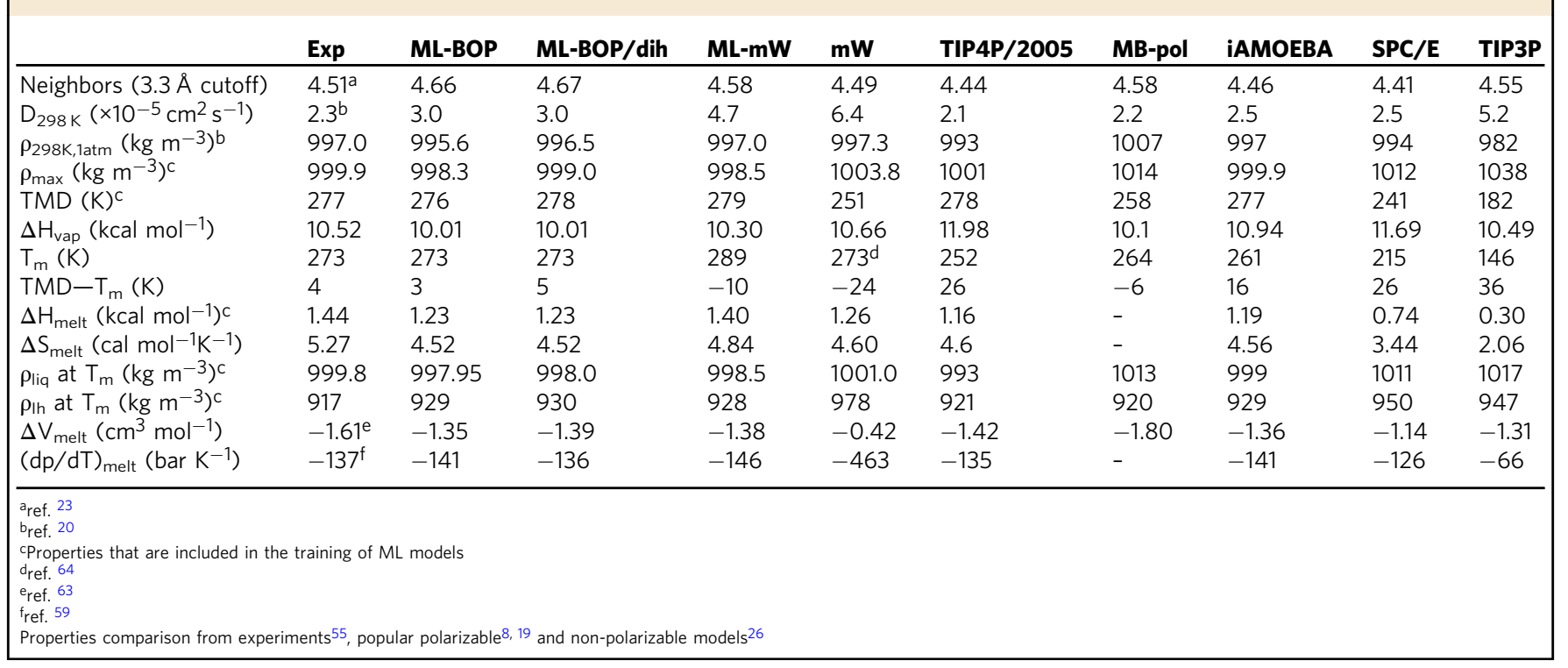

Table 6 Mean enthalpy and free-energy of various ice polytypes predicted by ML-BOP dih $_{\text {}}$

\begin{tabular}{|llll|} 
Stacking & $\begin{array}{l}\text { Mean enthalpy } \\
\text { (eV/molecule) }\end{array}$ & $\begin{array}{l}\text { Free energy, G } \\
\text { (eV/molecule) }\end{array}$ & $\begin{array}{l}\text { G - Glh } \\
\text { (meV/molecule) }\end{array}$ \\
\hline$I_{C}(A B C A B C)$ & -0.39506 & -0.50768487 & 0.959 \\
$I_{h}(A B A B A B)$ & -0.39528 & -0.50864357 & 0.000 \\
$A B A B C B$ & -0.39526 & -0.50809176 & 0.552 \\
$A B A C B C$ & -0.39511 & -0.50790687 & 0.737 \\
$A B C A C B$ & -0.39509 & -0.50793255 & 0.711 \\
$A B C B A B$ & -0.39526 & -0.50808043 & 0.563 \\
ABCBCB & -0.39523 & -0.50810750 & 0.536 \\
\hline
\end{tabular}

The mean enthalpy and free-energy (eV/molecule) are computed at $260 \mathrm{~K}$. The free energy difference relative to the most stable hexagonal ice phase is also given

The HOGA algorithm is able to efficiently sample the highdimensional parameter space and arrive at an optimal set of $\mathrm{mW}$ parameters with an improved overall score for the properties listed in Table 1.

Origin of the improvement in the ML model performance. To elucidate the improvements of the ML-mW and ML-BOP models, we compare the pair-wise interaction energy curves of these two models with the original $\mathrm{mW}$ model (Fig. 3a). As seen in the energy curves representing only the 2-body interactions (solid line style), there are two notable differences as we go from $\mathrm{mW}$ to $\mathrm{ML}-\mathrm{mW}$ to ML-BOP. There is a progressive steepening of the repulsive wall at $r<2.7 \AA$, and the interaction cutoffs become shorter $(4.3 \AA$ to $4.0 \AA$ to $3.6 \AA$ ). A large increase in repulsive interaction can also be inferred from the $\sim 3.3$ times larger value of parameter $B$ (coefficient of the repulsive term) in the functional form of ML-mW vs. mW (Supplementary Table 1). Furthermore, in contrast to a previous study that $\mathrm{mW}$ has the shortest optimal interaction cutoff necessary for capturing the anomalous properties of water ${ }^{25}$, HOGA is able to find a model with a shorter cutoff that improves the original model. The shorter cutoff of ML-mW also contributes to its improved efficiency over $\mathrm{mW}$ (Table 2). ML-mW has a $3^{\circ}$ deviation (left shift of minimum in Fig. 3b) from the ideal tetrahedral angle of $109.47^{\circ}$ (in $\mathrm{mW}$ ), and has a larger 3-body energy penalty for interatomic angles $\theta>130^{\circ}$ but a smaller penalty for $\theta<70^{\circ}$. The dashed and dotted cross marks in Fig. $3 \mathrm{~b}$ mark the interatomic angles $\left(\sim 37^{\circ}, \sim 72^{\circ}, \sim 155^{\circ}\right)$ at which the dashed and dotted energy curves in (a) are evaluated. The overall effect of bond order in the two models appears similar. We note that there have been prior efforts by Molinero and co-workers at improving the parameterization of the $\mathrm{mW}$ model using relative entropy minimization $^{26}$ (REM) as well as using uncertainty quantification ${ }^{25}$ (UQ). Both of these studies provide useful insights into the effect of model parameters on system properties. Note that while the search spaces in UQ were localized around the already optimized $\mathrm{mW}$ set, the parameter search in the REM procedure was global. In both the cases, the overall performance of those reparameterized models were found to be poorer when compared to the original $\mathrm{mW}$. In the present case, the performance improvements in ML-mW arise from a drastic deviation of potential parameters from the already optimized $\mathrm{mW}$ parameter set. This signifies the effectiveness of HOGA in navigating the high-dimensional parameter space and arriving at a set of optimal parameters that outperforms other optimization techniques such as REM and UQ.

Although both ML-mW and ML-BOP have quantitively improved the description of liquid density anomaly as well as the density of ice in $\mathrm{mW}$, only ML-BOP (in fact out of all currently existing water models) is able to capture the correct ordering and the relative temperature difference between the melting point and TMD of water. A major difference is the use of explicit cutoff by the Tersoff functional form (ML-BOP models) as against the implicit cutoff functions employed by StillingerWeber form (mW models). An explicit cutoff function provides the flexibility to modify the tail portion of the pair interaction energy curve independent of the rest of features such as the repulsive wall, location and depth of minimum, etc. (see inset of Fig. 3c). As the ML-BOP cutoff $(R+D)$ becomes smaller, while keeping the switching distance $(R-D)$ fixed, the relative separation between the melting point (cross marks) and TMD (vertical dotted lines) reduces and their ordering eventually flips (Fig. 3c). We further note that the tail portion of the interaction energy curve also has a strong influence on other properties including the liquid densities, ice densities close to the melting point, enthalpy of melting, diffusion coefficients, etc., which exemplifies the challenge in simultaneously optimizing many properties (i.e., multi-objective) and the need of a ML workflow 
a

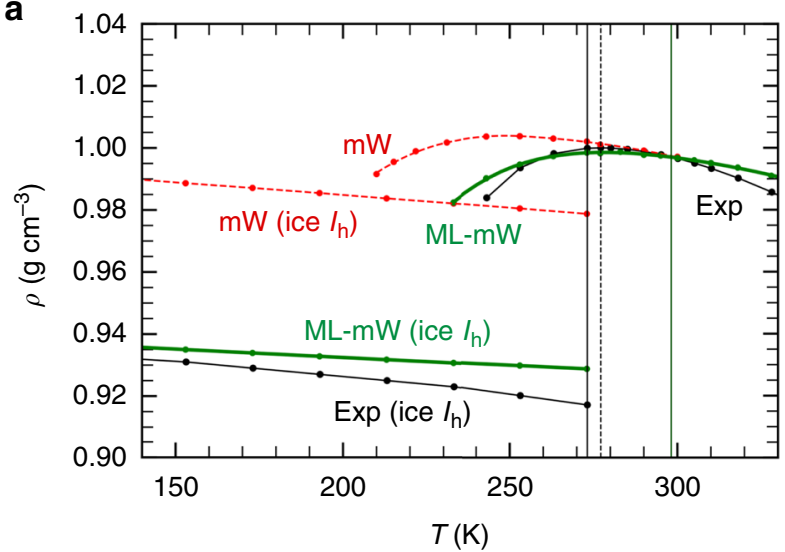

b

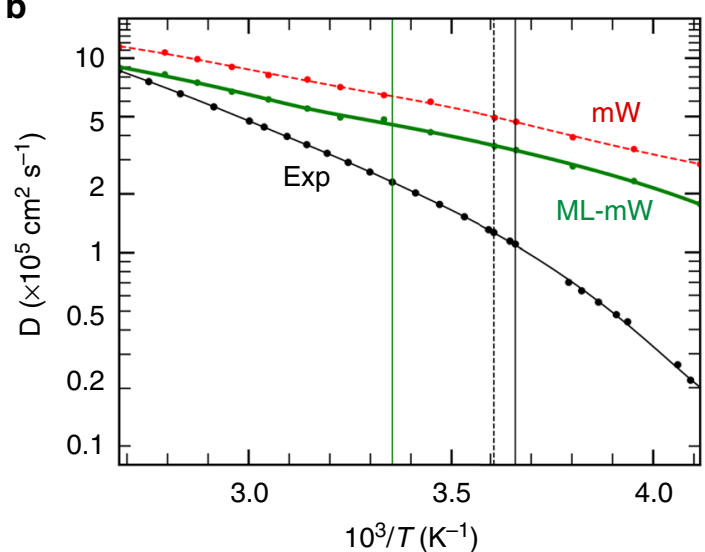

d

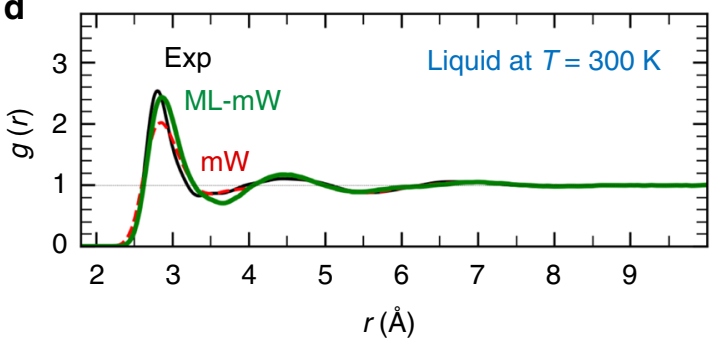

$r(\AA)$
C

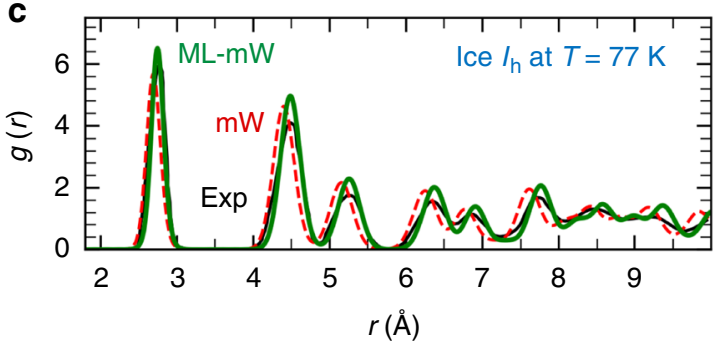

Fig. 2 Comparisons of the predicted properties of $M L-m W$ and the original $m W$ with experiments. In (a-b) the experimental (Exp) melting point $(T=273 \mathrm{~K})$, maximum density temperature $(T=277 \mathrm{~K})$, and room temperature $(T=298 \mathrm{~K})$ are vertical solid black, dotted black, and solid green lines, respectively. a Densities of ice and liquid water at pressure 1 bar. ML-mW melting point is $289 \pm 1 \mathrm{~K}$. b Diffusion coefficients of water over a wide temperature range. Radial distribution functions of $\mathbf{c}$ ice at $T=77 \mathrm{~K}$ and $\mathbf{d}$ liquid water at $T=300 \mathrm{~K}$. Table 3 has the optimized ML-mW parameters

in place of the local optimization based fitting procedures and/or driven by human intuition.

Simulations of ice nucleation in supercooled water. As a representative test case, we perform MD simulations on multimillion water molecules using ML-BOP models to understand at the molecular level homogeneous nucleation of supercooled water leading up to the formation and growth of grains of ice. Figure 4 summarizes the initial stages of nucleation leading up to the formation of polycrystalline ice for one such trajectory when water is slowly cooled from 275 to $210 \mathrm{~K}$ over $130.4 \mathrm{~ns}$ (cooling rate $\sim 0.5 \mathrm{~K} \mathrm{~ns}^{-1}$ ). Following the appearance of the first stable nuclei at $\sim 210 \mathrm{~K}$, the temperature was held at $210 \mathrm{~K}$ for a further $100 \mathrm{~ns}$ to study the nucleation and growth processes in this homogeneously nucleated water. Figure $4 \mathrm{a}$ shows the potential energy variation as a function of time during the cooling phase and constant temperature phase. We identify four distinct stages during the freezing process: a long quiescent time period of $\sim 130$ nanoseconds before the first nucleation events; a period of slow transformation with a limited number of nuclei ( 13 at $t=150 \mathrm{~ns}$, Fig. 4d); accelerated transformation driven by growth of a greater number of nuclei ( 185 at $200 \mathrm{~ns})$; and completion of grain growth to form a polycrystalline box of ice. Figure $4 \mathrm{~b}$ shows the corresponding snapshots during the initial quiescent period when the system explores the relatively flat energy landscape before entering the nucleation and growth period. The molecular level illustration is consistent with classical nucleation theory; the quiescent period is marked by pronounced fluctuations of many subcritical nuclei which rapidly form, break and reform in the supercooled liquid as shown in Fig. 4d. The post-quiescent period shown by MD snapshots in Fig. 4c is marked by formation of multiple stable nuclei which grow slowly followed by a rapid growth phase when the grains begin to percolate through the entire three-dimensional space. The completion of the growth phase is characterized by the formation of a polycrystalline ice with the nanoscopic grains separated by boundaries comprised of amorphous ice. A local structure analysis (see Methods) of the growing structure reveals that the grains are comprised of stacking disordered ice $\left(\mathrm{I}_{\mathrm{sd}}\right)$ i.e., randomly mixed alternating sheets of hexagonal and cubic ice (see Supplementary Figure 7 for the local structure). Figure $4 \mathrm{e}$ shows that the evolving ice structure becomes increasingly rich in $\mathrm{I}_{c}$ phase compared to the more stable $\mathrm{I}_{\mathrm{h}}$ phase with the ratio of cubic to hexagonal to be $\sim 1.85$ at the end of $t=350 \mathrm{~ns}$. The observed preference for cubic ice formation is consistent with multiple experimental results in the past including a recent X-ray diffraction study ${ }^{27}$ and CG simulations ${ }^{28}$ as well as atomistic simulations using forward-flux sampling technique ${ }^{29}$.

Nature of polycrystalline ice and transformation of stacking disordered ice to hexagonal ice. The final microstructure at 230 ns (Fig. 4c) is fine grained (average grain size 9300 water molecules) and is expected to anneal over long times (microsecond to seconds) to naturally observed larger grains. We slowly anneal the nanocrystalline sample by heating from 210 to $260 \mathrm{~K}$ over $100 \mathrm{~ns}$ and then hold the sample at $260 \mathrm{~K}$ until the grains coarsen. The polycrystalline sample evolves into a single grain at the end of 1 microsecond of simulation (Fig. 5a). The internal structure of the grains is ice $I_{s d}$, i.e., randomly mixed alternating sheets of hexagonal and cubic ice, comprised of stacking faults that evolve over time (Fig. $5 b$ ). The ice $\mathrm{I}_{\mathrm{sd}}$ structure observed in our simulations is rich in $\mathrm{I}_{c}$ phase compared to the more stable $\mathrm{I}_{\mathrm{h}}$ phase with the ratio of cubic to hexagonal being $\sim 2$ by $1200 \mathrm{~ns}$ (Fig. 4e).

The preference for ice $\mathrm{I}_{\mathrm{sd}}$ formation during nucleation is consistent with recent experiments and simulations ${ }^{27-30}$ but the 

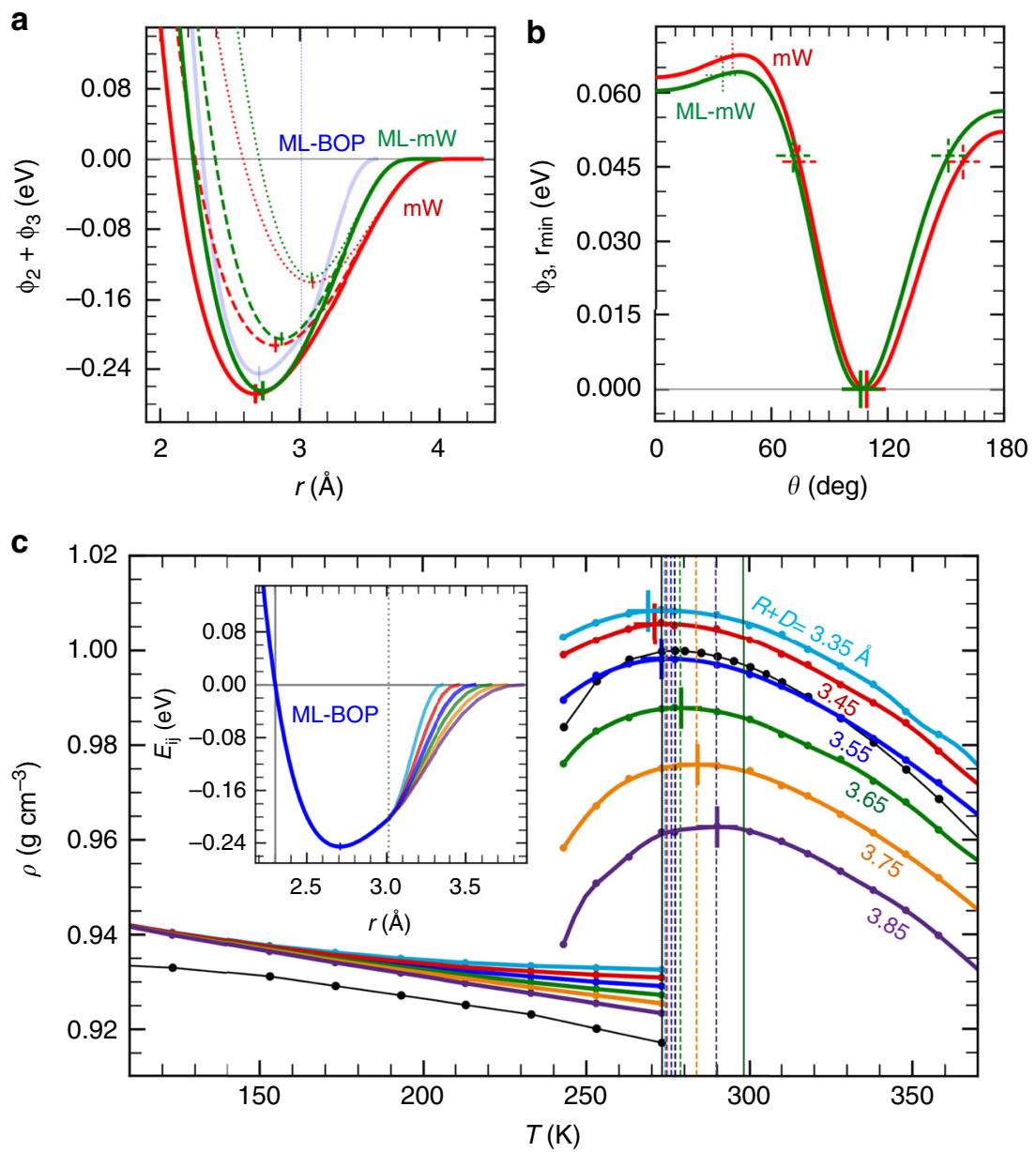

Fig. 3 Origin of the performance improvements in the ML-mW and ML-BOP models. a Solid line style curves compare the 2-body only interaction energy of $\mathrm{ML}-\mathrm{mW}, \mathrm{mW}$, and ML-BOP ( $\phi_{3}=0$ for the $\mathrm{mW}$ models and $\mathrm{b}_{i j}=1$ for ML-BOP). The vertical dotted line marks the Tersoff cutoff switching distance ( $R$ $-D$ ) in ML-BOP. Dashed and dotted line style curves show the pair interaction energy of $\mathrm{ML}-\mathrm{mW}$ and $\mathrm{mW}$ under the influence of a third atom, the addition of $\phi_{3}$. $\mathbf{b}$ 3-body energy term, $\phi_{3}$, evaluated at the minimum of the pair interaction energy curves, $r_{\min }$. Note that we first identify $r_{\text {min }}$ for each interatomic angle $\theta$ and then compute the corresponding $\phi_{3}$. The cross marks indicate the $\theta$ at which the interaction energy curves in (a) are evaluated. c The explicit cutoff function, $\mathrm{f}_{\mathrm{c}}$, in Tersoff provides a flexibility to independently modify the tail portion of the pair interaction energy curve (inset). This influences the relative separation between melting point (cross marks) and TMD (vertical dotted lines) in the temperature-dependent density plot

stacking disorder in polycrystalline ice has been much debated ${ }^{27}$. Kuhs et al. ${ }^{31}$ have analyzed neutron diffraction data and electron microscopy images to study the extent of stacking disorder in ice in the $170-190 \mathrm{~K}$ range. They tracked the evolution of cubicity as a function of time and note that the fraction of cubic stacking sequences is $\sim 0.5$. At temperatures $>180 \mathrm{~K}$, cubicity decreases slowly to approach pure $\mathrm{I}_{\mathrm{h}}$ after annealing over 10-12 h. Molinero and co-workers $^{28}$ have analyzed the structure of ice that crystallizes at $180 \mathrm{~K}$ and shown that the ratio of cubic to hexagonal stacking sequences is $\sim 2: 1$, which is similar to those found in our work. Indeed, more recent studies by Amaya et al. ${ }^{32}$ using femtosecond wide-angle $\mathrm{x}$-ray scattering confirm that ice formed by nanodroplets that freeze rapidly at timescales of the order of 1 microsecond indeed have much higher cubicity values $\sim 0.78 \pm 0.05$. This value is much higher than that reported by Kuhs et al. ${ }^{31}$. Nonetheless, these studies suggest that there can be a range of stacking disordered ice with different cubicity. The differences in the extent of stacking disorder were attributed to the differences in freezing temperatures ${ }^{33}$, the size of droplets (nanosized vs. micron sized) and the freezing rates $^{34}$ (microseconds vs. seconds) to name a few.

Capturing the energetic ordering and the subtle energetic differences between ice phases within a molecular model remains a major challenge. While the stable phase at weak undercooling is $\mathrm{I}_{\mathrm{h}}$, the ice phase that nucleates from supercooled water is, however, the stacking disordered ice. Free energy calculations performed by Molinero and co-workers ${ }^{30}$, using the $\mathrm{mW}$ model, show that the entropy of mixing of cubic and hexagonal layers makes stacking-disordered ice the stable phase for crystallites sizes up to 100,000 molecules. We note that the free energy cost of producing a growth fault in ice $\mathrm{I}_{\mathrm{h}}$ for the $\mathrm{mW}$ model is $\sim 15.3 \pm$ $2.3 \mathrm{~J} \mathrm{~mol}^{-1}(0.159 \pm 0.024 \mathrm{meV})$, which is consistent with the experimental value of $16.5 \pm 1.7 \mathrm{~J} \mathrm{~mol}^{-1} \quad(0.171 \pm 0.018 \mathrm{meV})$ reported by Hondoh et al. ${ }^{35}$. Depending on the experimental conditions and the method of sample preparation, there is a range of free energy or enthalpy reported for the transformation of stacking disordered ice to pure hexagonal ice. For example, Ghormley et al. ${ }^{36}$ report transformation of cubic to hexagonal crystals to be $\sim 22 \mathrm{~J} \mathrm{~mol}^{-1}(0.228 \mathrm{meV})$ in heating from 223 to $268 \mathrm{~K}$. Differential scanning calorimetry of transformation of cubic ice (prepared by rapid quenching of liquid water at $190 \mathrm{~K}$ ) to hexagonal ice report a slightly higher value $\sim 56 \mathrm{~J} \mathrm{~mol}^{-1}$ $(0.580 \mathrm{meV})$. On the other hand, McMillian et al. ${ }^{37}$ used calorimetry measurements and report a heat of transformation $\Delta \mathrm{H}=160 \mathrm{~J} \mathrm{~mol}^{-1}(1.658 \mathrm{meV})$ between 'cubic' and hexagonal ice. Likewise, Shilling et al. ${ }^{38}$ prepared amorphous ices by vapor 
a

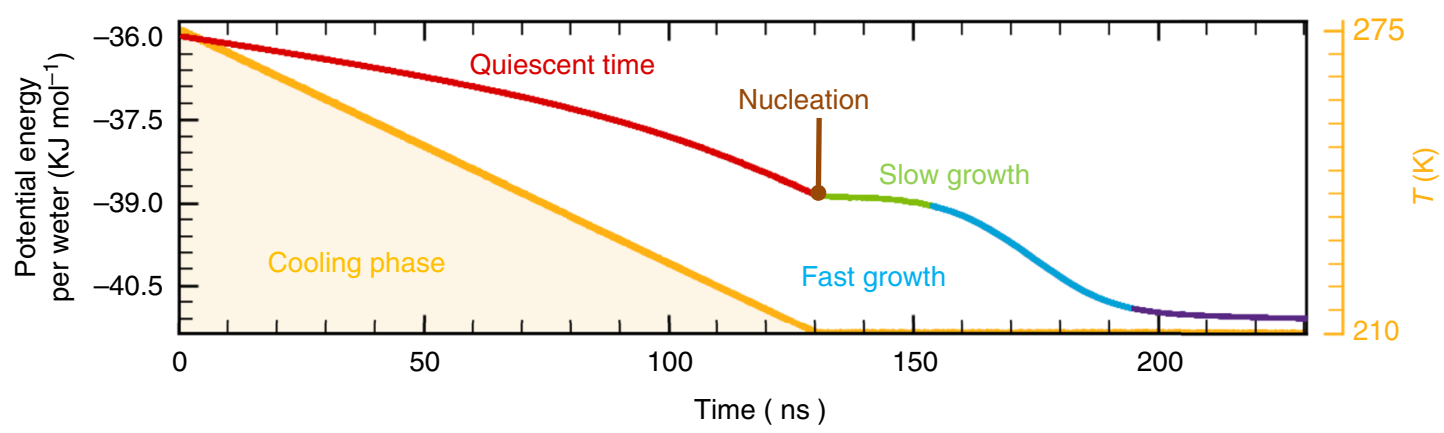

b
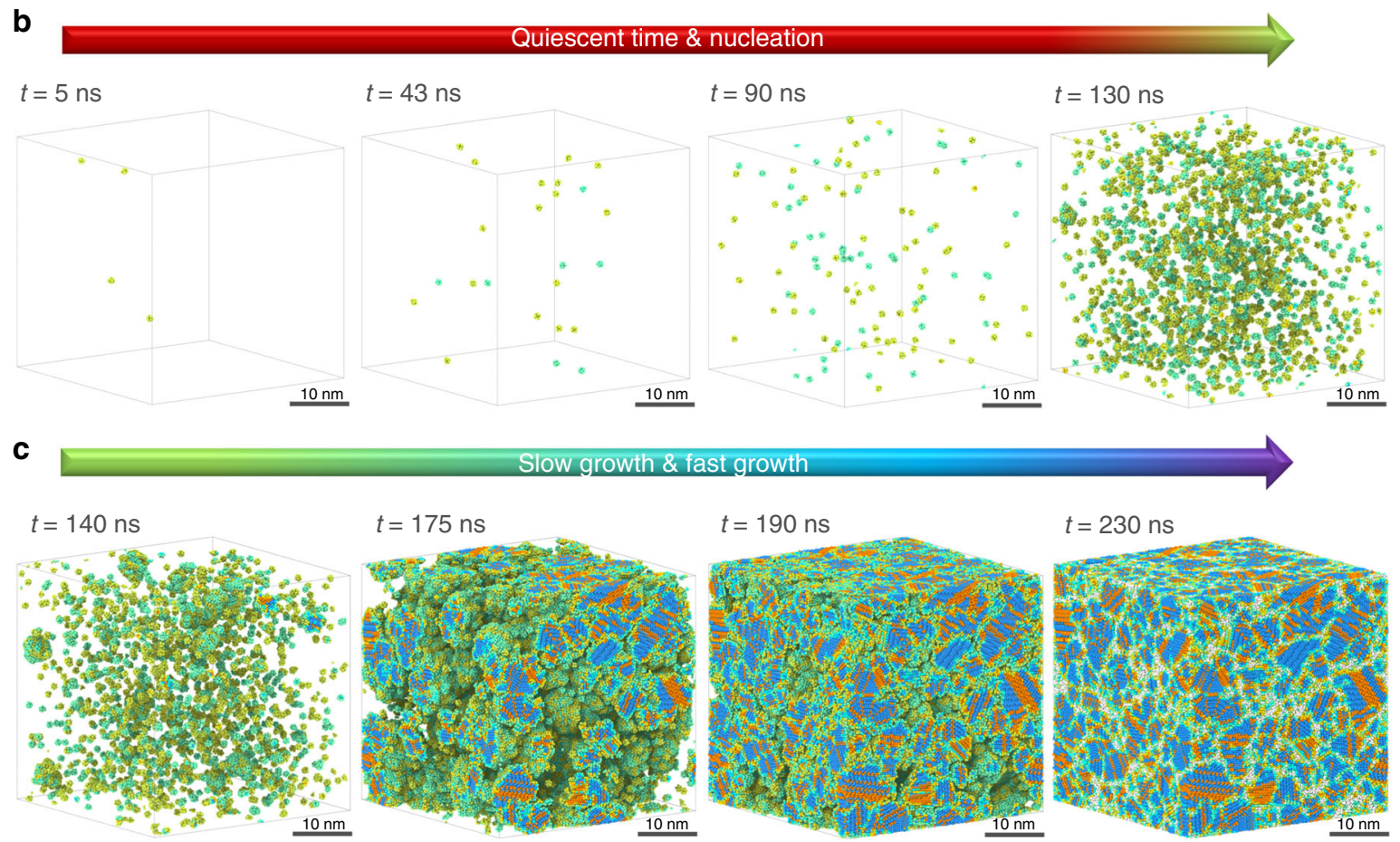

d

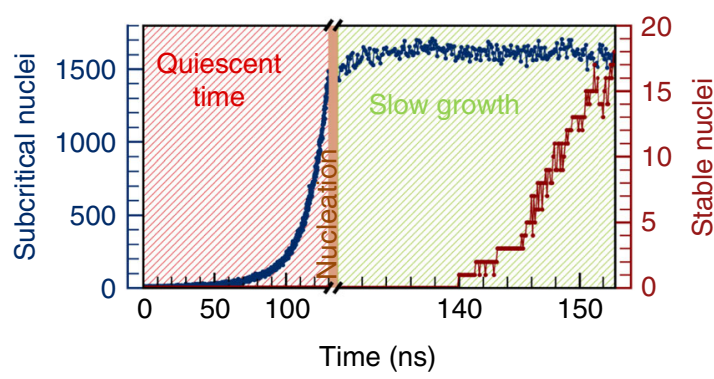

\section{.}

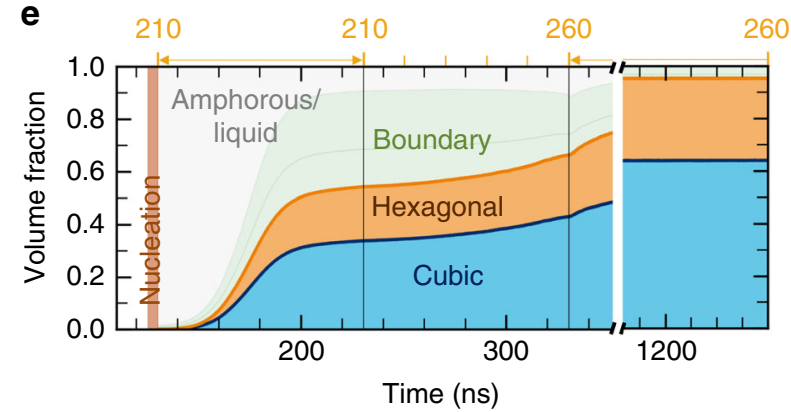

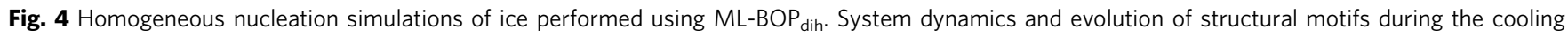
phase from homogeneous nucleation leading up to the grain boundary formation and grain growth (Supplementary Movie 1). a The total potential energy variation of the 2 million-water molecule system during the cooling phase from 275 to $210.5 \mathrm{~K}$ and at longer times when the system temperature is kept constant at $210.5 \mathrm{~K}$. We identify four distinct stages: an initial quiescent time shown by the red line when no nucleation event occurs; the nucleation followed by an initial slow transformation shown by the slow energy decreasing period in green; a fast transformation phase of the grains shown by the rapid decrease in potential energy in blue; and a plateauing of potential energy shown in purple marks the completion of the phase transformation. $\mathbf{b}$ The snapshots show the subcritical water nuclei during the long quiescent phase leading up to the nucleation. The first nucleation event for the 2 million-water system occurs at $t=130 \mathrm{~ns}$. Liquid water molecules are not shown for clarity. c MD simulation snapshots showing the various stages of grain growth and grain boundary during the post-nucleation stage. Blue, brown and green spheres represent cubic, hexagonal and amorphous ice, respectively. Liquid water is omitted for clarity. d The temporal evolution of the number of subcritical water nuclei (size $<100$ molecules) from the quiescent period and the initial appearance of stable nuclei during the post-nucleation stage. e The corresponding temporal evolution of the fraction of cubic and hexagonal ice 
a

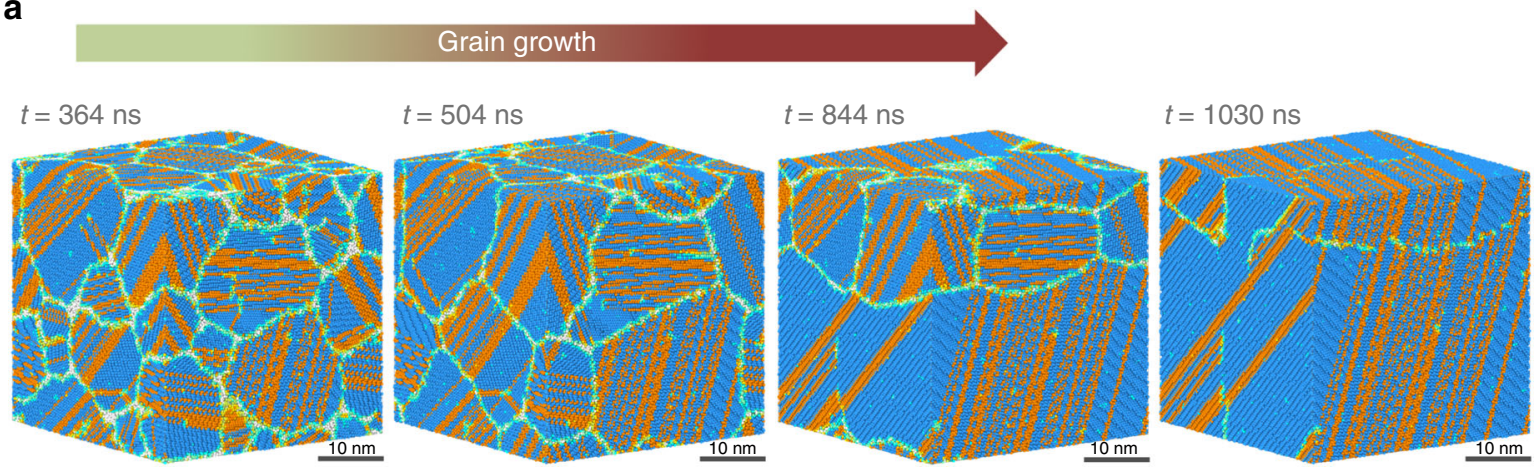

\section{b Evolution of stacking faults}
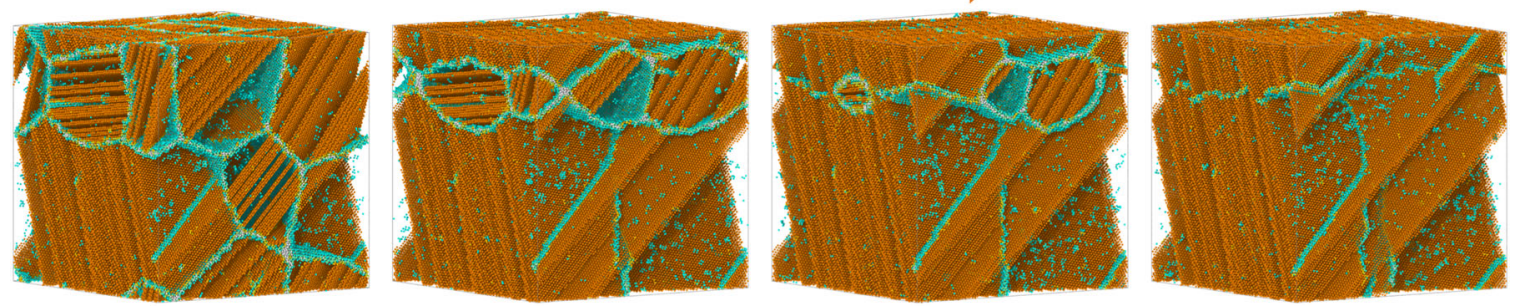

C
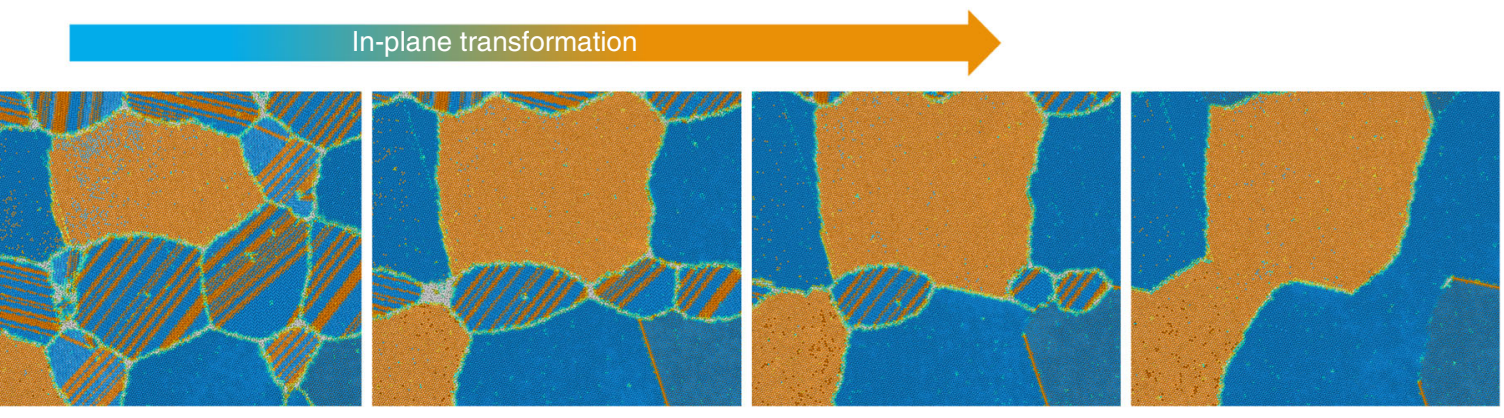

Fig. 5 Post-nucleation ice grain growth simulations performed using ML-BOP ${ }_{\text {dih. }}$. System dynamics and evolution of structural motifs of post-nucleation phase after the slow heating from 210 to $260 \mathrm{~K}$. a Snapshots from simulations showing the grain growth process of nanosized grains at $260 \mathrm{~K}$ evolving into a single grain. (see Supplementary Movie 2 for a zoom-in view) b Snapshots from simulations (Supplementary Movie 3) showing the time evolution of hexagonal layers in stacking disordered ice. Cubic type molecules are not shown for clarity. Note that the ML-BOP model considers only nearest neighbor interactions but is able to reproduce the random stacking-disorder which is consistent with experimental observations ${ }^{31}$. c Snapshots from simulations (Supplementary Movie 4) showing the in-plane transformation between cubic and hexagonal layers in stacking disordered ice, viewed along the direction perpendicular to the basal plane of the largest stacking ice grain in the system

deposition at $90 \mathrm{~K}$ and transformed them to stacking disordered ice by heating up to about $160 \mathrm{~K}$. They report a free energy change of $155 \pm 30 \mathrm{~J} \mathrm{~mol}^{-1}(1.606 \pm 0.31 \mathrm{meV})$ for transforming $I_{s d}$ to $I_{h}$. This value is much higher than that reported by Ghormley et al. ${ }^{36}$. Differential thermal analysis also suggests heat release of similar order when $I_{c}$ transforms to more thermodynamically stable $\mathrm{I}_{\mathrm{h}}{ }^{39}$. Note that the $\mathrm{mW}$ predicted free energy is lower than the experimental values of Shilling et al..$^{38}$ and McMillian et al. ${ }^{37}$. On the other hand, recent ab initio studies also suggest a thermodynamic preference for $\mathrm{I}_{\mathrm{h}}$ compared to $\mathrm{I}_{\mathrm{c}}(\sim 1.4$ meV per $\mathrm{H}_{2} \mathrm{O}$ arising from the difference in anharmonicity between cubic and hexagonal ice $)^{14}$. While DFT-PBE may not be the best method for estimating the energetics of ice, this high free energy difference between $I_{c}$ and $I_{h}$ cannot be captured by nearest neighbor interactions as is the case in ML-BOP, ML-mW and $\mathrm{mW}$. We, therefore, introduce an additional four-body term to the ML-BOP in the form of on-the-fly dihedrals model and retrained this $\mathrm{ML}-\mathrm{BOP}_{\mathrm{dih}}$ model by including the average energy difference between cubic and hexagonal ice (reported in ref. ${ }^{14}$ using DFT-PBE) in the training data set. This 4-body term essentially captures the energetics difference provided by the PBE input data in ref. ${ }^{14}$. One can retrain the 4-body term (Eqs. 8-10) if new improved ab initio data becomes available.

To test the thermodynamic preference of our new ML-BOP ${ }_{\text {dih }}$ model, we calculate the free energies of various ice phases (Fig. 6a) within the quasi-harmonic approximation (see Methods). Our model is able to capture the temperature-dependent stability of cubic, stacking disordered and hexagonal phases; hexagonal is the most stable phase and is $\sim 1 \mathrm{meV}$ per molecule lower than the metastable cubic phase at $260 \mathrm{~K}$ (Table 6). Despite $I_{h}$ being energetically preferred compared to $I_{s d}$, we do not observe a transformation to a pure hexagonal phase even after $1.3 \mu$ s of simulations possibly due to sufficiently large activation barrier. Indeed, climbing image Nudged Elastic Band (CI-NEB) calculations within the framework of ML-BOP ${ }_{\text {dih }}$ show that the energetic barrier associated with elimination of a stacking fault plane in hexagonal ice is $\sim 170 \mathrm{meV}$ (Fig. 6b). The atomic-scale pathway governing the transformation of a representative $\mathrm{I}_{\mathrm{sd}}$ with $A B C B A B$ stacking to $I_{h}(A B A B A B)$ is shown in Fig. 6b. The sliding of the molecules in the $\mathrm{C}$-plane to their respective A-plane positions entails a range of concerted molecular motions involving stretching/rotation of hydrogen bonds. These 
a

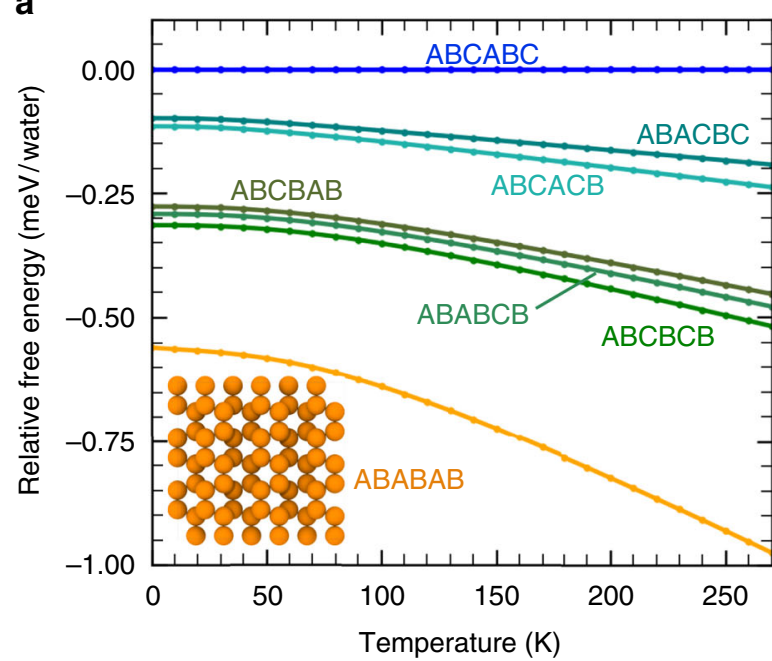

$A B C A B C$

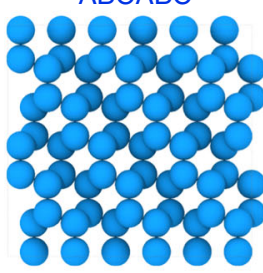

$A B C B A B$

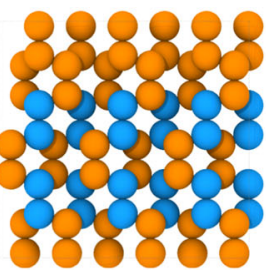

ABACBC

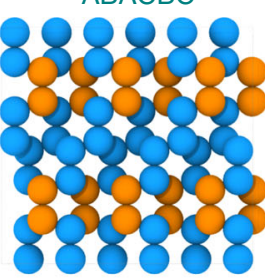

$A B A B C B$

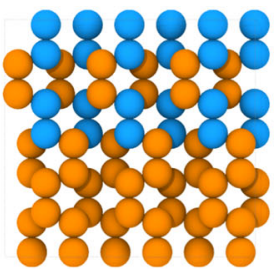

$A B C A C B$

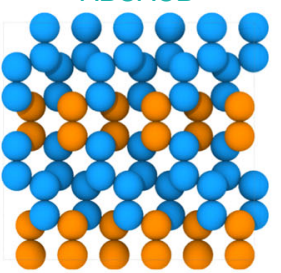

ABCBCB

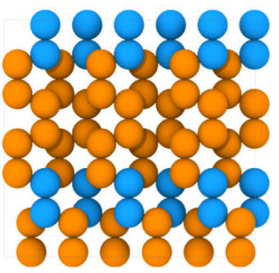

b
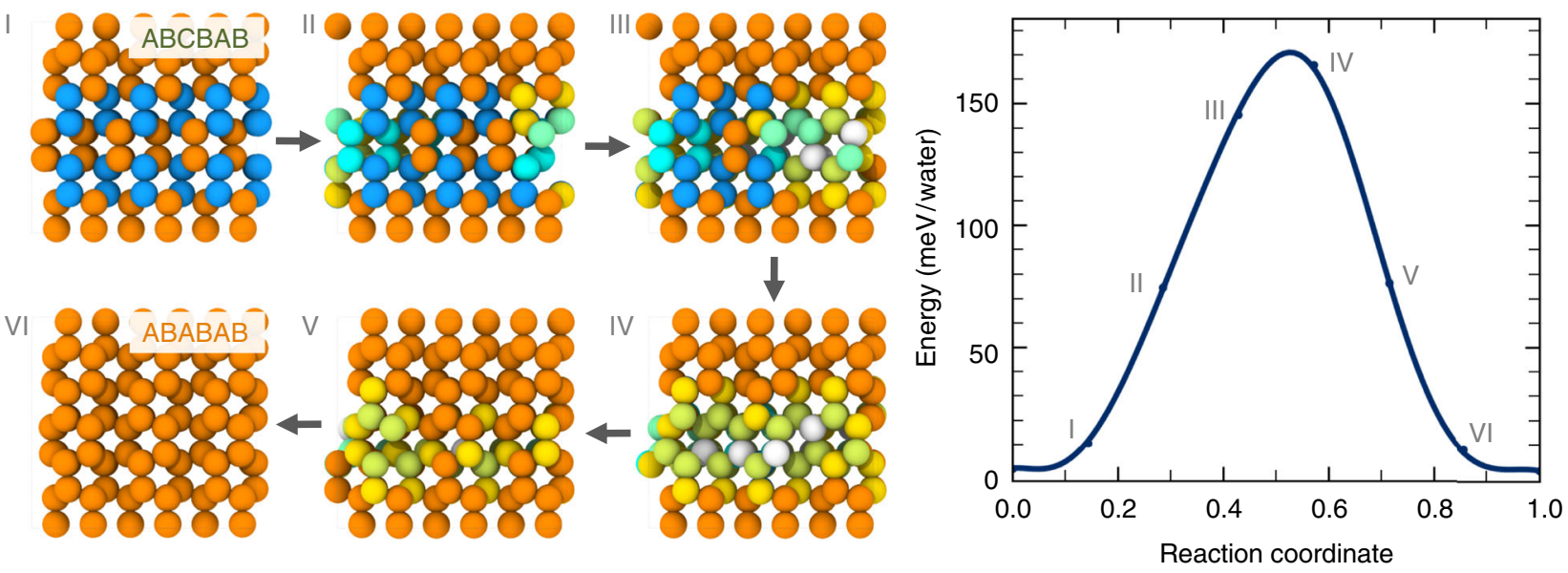

Fig. 6 Thermodynamics of various ice phases and activation barrier computed using ML-BOP dih. a Free energies of various stacked disorder ice phases, $I_{c}$ and $I_{h}$ at different temperatures, relative to $I_{c}$. Molecular configurations of the various phases are also provided. $\mathbf{b}$ Molecular pathway, and the associated energy barrier for elimination of a stacking fault plane in $I_{h}$. Selected configurations along the pathway are shown on the left, while potential energy along this preferred pathway (obtained by $\mathrm{CI}-\mathrm{NEB}$ calculations in the framework of $\mathrm{ML}-\mathrm{BOP}_{\text {dih }}$ ) is shown on the right. All the molecules are colored based on their local coordination using the same scheme as Fig. 4

coordinated movements result in localized strain in the vicinity of the stacking fault plane and underlie the activation barrier $(\sim 170 \mathrm{meV})$ associated with elimination of the fault. Thermal fluctuations at $260 \mathrm{~K}(\mathrm{kT} \sim 22 \mathrm{meV})$ are sufficiently small to preclude observation of the $I_{s d} \rightarrow I_{h}$ transformation within $\mu$ s timescales. In-plane $\mathrm{I}_{\mathrm{sd}} \rightarrow \mathrm{I}_{\mathrm{h}}$ transitions have much lower barriers $(\sim 5 \mathrm{meV}$ per water) and are frequently observed at MD timescales (see Fig. 5c and Supplementary Movie 3, 4). Indeed, this behavior is consistent with the partial dislocation mechanism proposed by Hondoh et al. ${ }^{40}$. It is also worth noting that the hexagonal ice becomes thermodynamically more favorable as we approach the melting point; hence the free energy difference between $I_{h}$ and $I_{c}$ is expected to increase (as shown in Fig. 6a). The different stacking-disordered ice configurations have much smaller free energy difference $(<0.2 \mathrm{meV}$ per water), which can explain the presence of random stacking disorder observed in previous experiments $^{41}$ and simulations ${ }^{27,28,31,41-43}$.

In summary, we introduced a machine learning strategy to train CG models, namely ML-BOP, ML-BOP ${ }_{\text {dih }}$ and ML-mW, for water simulations. As proof-of-principle, we use the developed ML CG models (ML-BOP and ML-BOP ${ }_{\text {dih }}$ ) to elucidate the mesoscale mechanism of ice grain formation and growth from supercooled water. In light of the accuracy and speed of our ML potential models, we foresee their wide usage in problems including phase transitions, homogeneous and heterogeneous nucleation, interfacial properties, co-existent regimes, and mechanical behavior, all at system sizes and times inaccessible to current popular models such as the TIP5P and TIP4P models.

\section{Methods}

Molecular dynamics simulations. Using the final ML-BOP and ML-BOP ${ }_{\text {dih }}$ potentials, we perform massively parallel, long-time MD simulations of supercooled water to study the sequence of steps from nucleation to grain formation and coarsening. We start with a simulation cell containing 2,048,000 molecules of water at $275 \mathrm{~K}$. Simulations with a larger sized cell containing $\sim 8$ million water molecules are also performed. In both the cases, the structure is minimized and equilibrated for $100 \mathrm{ps}$ at $275 \mathrm{~K}$ in an NPT ensemble. Subsequently, the liquid water is cooled down to $200 \mathrm{~K}$ over $150 \mathrm{~ns}$ under isobaric conditions. We observe the first stable nuclei at $\sim 210 \mathrm{~K}$. Consequently, we stop the cooling at $210 \mathrm{~K}$ and hold the supercooled liquid at $210 \mathrm{~K}$ for $100 \mathrm{~ns}$. Following nucleation and growth of nanocrystalline ice grains at this temperature, we anneal the structure to $260 \mathrm{~K}$ at a rate of $0.5 \mathrm{~K} \mathrm{~ns}^{-1}$ and then hold it at $260 \mathrm{~K}$ beyond a microsecond to study the temporal evolution of grains.

Identification of ice polytypes and grains. Hexagonal $\left(I_{h}\right)$, cubic $\left(I_{c}\right)$, and amorphous/liquid phases of ice are determined using a structure identification algorithm ${ }^{44}$ implemented in the visualization software OVITO ${ }^{45}$. The molecules are color coded according to their local environment (see Supplementary Figure 7). A feature detection algorithm based on image processing and unsupervised 
machine learning (clustering) techniques ${ }^{46}$ is developed to identify individual grains and their size distribution. The procedure involves voxelization ( $5 \AA$ bin), contrasting filters, thresholding, DBSCAN clustering ${ }^{47}$, refinement, and positionbased reverse mapping. All nearest neighbor searches are performed using a periodic $\mathrm{k}-\mathrm{d}$ tree. This grain identification procedure accurately identifies small and large grains that are often irregularly shaped.

Energy barrier calculations of stacking faults. The activation energy associated with the elimination of stacking fault plane in hexagonal ice is computed using Climbing Image Nudged Elastic Band (CI-NEB) calculations within the framework of ML-BOP ${ }_{\text {dih }}$ as implemented in LAMMPS ${ }^{48-50}$. For these calculations, the computational supercell consists of 6 layers (432 water molecules), with 72 molecules in the stacking fault plane.

Free energy calculations. Free energies of the cubic, hexagonal, and stacking disorder ice polytypes are computed within the quasi-harmonic approximation ${ }^{51}$ using Phonopy ${ }^{52}$ to account for variation of phonon modes due to thermal expansion. For each ice phase, we first optimize the geometry of the unit cell in the framework of $\mathrm{ML}-\mathrm{BOP}_{\mathrm{dih}}$ until the energy difference between consecutive steps is $<10^{-4} \mathrm{eV}$, and the atomic forces are within $10^{-3} \mathrm{eV} \AA^{-1}$. Next, we determine the changes in volume owing to thermal expansion using $1 \mathrm{~ns}$ long MD simulations at zero pressure and desired temperature. At each volume, phonon frequencies and related vibrational properties are computed via finite displacement approach using sufficiently large supercells $(\sim 88 \AA \times 70 \AA \times 66 \AA)$ and displacement of $0.015 \AA$. The computed volume-dependence of phonon frequencies are then used to compute vibrational contribution to free energy at each temperature.

Ice-liquid surface tension calculations. The liquid-ice surface tension is calculated using the mold integration method ${ }^{53}$. The well depth is chosen to be $10 \mathrm{meV}$ based the value chosen for the original mW model ${ }^{54}$. The system consists of 1600 wells and a total of 6000 water molecules. The temperature for this calculation was chosen to be the freezing temperature for each of the respective models. The slab is 2 unit cells in thickness in the Z-direction which is the interfacial direction. To obtain the free energy vs well radius curve, a series of thermodynamic integrations are performed by incrementing the well radius by $0.1 \AA$ increments. This covers a range from $0.4 \AA$ up to $1.2 \AA$. These values were used to create a linear curve that could be used to extrapolate back to the optimal well radius to obtain the correct value for the surface tension. This is in line with the procedure outlined in ref. ${ }^{53}$. After the curve is obtained, the optimal well depth for both systems is determined by running a simulation at each well radius and monitoring the crystallinity of the system. Each simulation is run for 10 ns. Once an approximate range is identified a set of intermediate radius values is examined to find the optimal well radius. We first benchmarked our procedure for the original $\mathrm{mW}$ and validate that the iceliquid surface tension was $35 \mathrm{~mJ} / \mathrm{m}^{2}$ consistent with that reported in refs. ${ }^{53,54}$. For the ML-BOP and ML-BOP dih it is found to be roughly $0.65 \mathrm{~A}$ while for the MLMW it is found to be $0.45 \AA$, which yields the values in Table 4 .

Code availability. Code and workflow developed in this study are available from the authors upon reasonable request.

\section{Data availability}

The data that support the findings of this study are available from the authors upon reasonable request.

Received: 5 July 2018 Accepted: 19 December 2018

Published online: 22 January 2019

\section{References}

1. Liu, J., Nicholson, C. E. \& Cooper, S. J. Direct measurement of critical nucleus size in confined volumes. Langmuir 23, 7286-7292 (2007).

2. Faria, S. H., Weikusat, I. \& Azuma, N. The microstructure of polar ice. Part I: Highlights from ice core research. J. Struct. Geol. 61, 2-20 (2014).

3. Sosso, G. C. et al. Crystal nucleation in liquids: open questions and future challenges in molecular dynamics simulations. Chem. Rev. 116, 7078-7116 (2016).

4. Mahoney, M. W. \& Jorgensen, W. L. A five-site model for liquid water and the reproduction of the density anomaly by rigid, nonpolarizable potential functions. J. Chem. Phys. 112, 8910-8922 (2000).

5. Abascal, J. L. F. \& Vega, C. A general purpose model for the condensed phases of water: TIP4P/2005. J. Chem. Phys. 123, 234505 (2005).

6. Jorgensen, W. L. \& Tirado-Rives, J. Potential energy functions for atomic-level simulations of water and organic and biomolecular systems. Proc. Natl Acad. Sci. USA 102, 6665-6670 (2005).
7. Hadley, K. R. \& McCabe, C. Coarse-grained molecular models of water: a review. Mol. Simul. 38, 671-681 (2012).

8. Reddy, S. K. et al. On the accuracy of the MB-pol many-body potential for water: Interaction energies, vibrational frequencies, and classical thermodynamic and dynamical properties from clusters to liquid water and ice. J. Chem. Phys. 145, 194504 (2016).

9. Ren, P. \& Ponder, J. W. Polarizable atomic multipole water model for molecular mechanics simulation. J. Phys. Chem. B 107, 5933-5947 (2003).

10. Molinero, V. \& Moore, E. B. Water modeled as an intermediate element between carbon and silicon. J. Phys. Chem. B 113, 4008-4016 (2009).

11. Agarwal, M., Alam, M. P. \& Chakravarty, C. Thermodynamic, diffusional, and structural anomalies in rigid-body water models. J. Phys. Chem. B 115, 6935-6945 (2011).

12. Tersoff, J. New empirical approach for the structure and energy of covalent systems. Phys. Rev. B 37, 6991-7000 (1988).

13. Stuart, S. J., Tutein, A. B. \& Harrison, J. A. A reactive potential for hydrocarbons with intermolecular interactions. J. Chem. Phys. 112, 6472-6486 (2000).

14. Engel, E. A., Monserrat, B. \& Needs, R. J. Anharmonic nuclear motion and the relative stability of hexagonal and cubic ice. Phys. Rev. X 5, 021033 (2015).

15. Plimpton, S. Fast Parallel Algorithms for Short-Range Molecular Dynamics. J. Comput. Phys. 117, 1-19 (1995).

16. Mitchell M. An Introduction to Genetic Algorithms. (MIT Press, Cambridge, MA, 1996).

17. Nelder, J. A. \& Mead, R. A simplex method for function minimization. Comput. J. 7, 308-313 (1965).

18. Vega, C., Abascal, J. L. F., Conde, M. M. \& Aragones, J. L. What ice can teach us about water interactions: a critical comparison of the performance of different water models. Faraday Discuss. 141, 251-276 (2009).

19. Wang, L.-P. et al. Systematic improvement of a classical molecular model of water. J. Phys. Chem. B 117, 9956-9972 (2013).

20. Holz, M., Heil, S. R. \& Sacco, A. Temperature-dependent self-diffusion coefficients of water and six selected molecular liquids for calibration in accurate 1H NMR PFG measurements. Phys. Chem. Chem. Phys. 2, 4740-4742 (2000).

21. Kuo, I. F. W. et al. Liquid water from first principles: investigation of different sampling approaches. J. Phys. Chem. B 108, 12990-12998 (2004).

22. Morrone, J. A. \& Car, R. Nuclear quantum effects in water. Phys. Rev. Lett. 101, 017801 (2008).

23. Skinner, L. B., Benmore, C. J., Neuefeind, J. C. \& Parise, J. B. The structure of water around the compressibility minimum. J. Chem. Phys. 141, 214507 (2014).

24. Soper, A. K. The radial distribution functions of water as derived from radiation total scattering experiments: is there anything we can say for sure? Int. Sch. Res. Not. 2013, e279463 (2013).

25. Jacobson, L. C., Kirby, R. M. \& Molinero, V. How short is too short for the interactions of a water potential? exploring the parameter space of a coarsegrained water model using uncertainty quantification. J. Phys. Chem. B 118, 8190-8202 (2014).

26. Lu, J., Qiu, Y., Baron, R. \& Molinero, V. Coarse-graining of TIP4P/2005, TIP4P-Ew, SPC/E, and TIP3P to monatomic anisotropic water models using relative entropy minimization. J. Chem. Theory Comput. 10, 4104-4120 (2014).

27. Malkin, T. L., Murray, B. J., Brukhno, A. V., Anwar, J. \& Salzmann, C. G. Structure of ice crystallized from supercooled water. Proc. Natl Acad. Sci. USA 109, 1041-1045 (2012).

28. Moore, E. B. \& Molinero, V. Is it cubic? Ice crystallization from deeply supercooled water. Phys. Chem. Chem. Phys. 13, 20008-20016 (2011).

29. Haji-Akbari, A. \& Debenedetti, P. G. Direct calculation of ice homogeneous nucleation rate for a molecular model of water. Proc. Natl Acad. Sci. USA 112 10582-10588 (2015).

30. Lupi, L. et al. Role of stacking disorder in ice nucleation. Nature 551, 218 (2017).

31. Kuhs, W. F., Sippel, C., Falenty, A. \& Hansen, T. C. Extent and relevance of stacking disorder in "ice Ic". Proc. Natl Acad. Sci. USA 109, 21259 (2012).

32. Amaya, A. J. et al. How cubic can ice be? J. Phys. Chem. Lett. 8, 3216-3222 (2017).

33. Johnston, J. C. \& Molinero, V. Crystallization, melting, and structure of water nanoparticles at atmospherically relevant temperatures. J. Am. Chem. Soc. 134, 6650-6659 (2012)

34. Moore, E. B. \& Molinero, V. Ice crystallization in water's "no-man's land". J. Chem. Phys. 132, 244504 (2010).

35. Hondoh, T., Itoh, T., Amakai, S., Goto, K. \& Higashi, A. Formation and annihilation of stacking faults in pure ice. J. Phys. Chem. 87, 4040-4044 (1983).

36. Ghormley, J. A. Enthalpy changes and heat-capacity changes in the transformations from high-surface-area amorphous ice to stable hexagonal ice. J. Chem. Phys. 48, 503-508 (1968). 
37. McMillan, J. A. \& Los, S. C. Vitreous ice: irreversible transformations during warm-up. Nature 206, 806 (1965).

38. Shilling, J. E. et al. Measurements of the vapor pressure of cubic ice and their implications for atmospheric ice clouds. Geophys. Res. Lett. 33, L17801 (2006).

39. Murray, B. J., Knopf, D. A. \& Bertram, A. K. The formation of cubic ice under conditions relevant to Earth's atmosphere. Nature 434, 202 (2005).

40. Hondoh, T. Dislocation mechanism for transformation between cubic ice Ic and hexagonal ice Ih. Philos. Mag. 95, 3590-3620 (2015).

41. Malkin, T. L. et al. Stacking disorder in ice I. Phys. Chem. Chem. Phys. 17, 60-76 (2015).

42. Hansen, T., Falenty, A. \& Kuhs, W. Modelling ice Ic of different origin and stacking-faulted hexagonal ice using neutron powder diffraction data. Spec. Publ. - R. Soc. Chem. 311, 201 (2006).

43. Correction for Malkin. et al. Structure of ice crystallized from supercooled water. Proc. Natl Acad. Sci. USA 109, 4020 (2012).

44. Maras, E., Trushin, O., Stukowski, A., Ala-Nissila, T. \& Jónsson, H. Global transition path search for dislocation formation in $\mathrm{Ge}$ on $\mathrm{Si}(001)$. Comput. Phys. Commun. 205, 13-21 (2016).

45. Stukowski, A. Visualization and analysis of atomistic simulation data with OVITO-the Open Visualization Tool. Model. Simul. Mater. Sci. Eng. 18, 015012 (2010).

46. Gan G., Ma C., Wu J. Data Clustering: Theory, Algorithms, and Applications. (Siam, Philadelphia, PA, 2007).

47. Ester, M., Kriegel, H.-P., Sander, J. \& Xu, X. A density-based algorithm for discovering clusters in large spatial databases with noise. (ed^(eds). (AAAI Press, 1996).

48. Henkelman, G. \& Jónsson, H. Improved tangent estimate in the nudged elastic band method for finding minimum energy paths and saddle points. J. Chem. Phys. 113, 9978-9985 (2000).

49. Henkelman, G., Uberuaga, B. P. \& Jónsson, H. A climbing image nudged elastic band method for finding saddle points and minimum energy paths. J. Chem. Phys. 113, 9901-9904 (2000).

50. Nakano, A. A space-time-ensemble parallel nudged elastic band algorithm for molecular kinetics simulation. Comput. Phys. Commun. 178, 280-289 (2008).

51. Plata, J. J. et al. An efficient and accurate framework for calculating lattice thermal conductivity of solids: AFLOW-AAPL Automatic Anharmonic Phonon Library. npj Comput. Mater. 3, 45 (2017).

52. Togo, A. \& Tanaka, I. First principles phonon calculations in materials science. Scr. Mater. 108, 1-5 (2015).

53. Espinosa, J. R., Vega, C. \& Sanz, E. The mold integration method for the calculation of the crystal-fluid interfacial free energy from simulations. J. Chem. Phys. 141, 134709 (2014).

54. Qiu, Y., Lupi, L. \& Molinero, V. Is Water at the Graphite Interface Vapor-like or Ice-like? J. Phys. Chem. B 122, 3626-3634 (2018).

55. CRC Handbook of Chemistry and Physics: A Ready-reference Book of Chemical and Physical Data, 85. ed edn. CRC Press (2004).

56. Gillen, K. T., Douglass, D. C. \& Hoch, M. J. R. Self-diffusion in liquid water to $-31^{\circ} \mathrm{C}$. J. Chem. Phys. 57, 5117-5119 (1972).

57. Narten, A. H., Venkatesh, C. G. \& Rice, S. A. Diffraction pattern and structure of amorphous solid water at 10 and $77^{\circ} \mathrm{K}$. J. Chem. Phys. 64, 1106-1121 (1976).

58. Chickos, J. S.Jr. \& AcreeW. E. Enthalpies of Sublimation of Organic and Organometallic Compounds. 1910-2001. J. Phys. Chem. Ref. Data 31, 537-698 (2002).

59. Vega, C. \& Abascal, J. L. F. Simulating water with rigid non-polarizable models: a general perspective. Phys. Chem. Chem. Phys. 13, 19663-19688 (2011).

60. Ketcham, W. M. \& Hobbs, P. V. An experimental determination of the surface energies of ice. Philos. Mag. 19, 1161-1173 (1969).

61. Handel, R., Davidchack, R. L., Anwar, J. \& Brukhno, A. Direct calculation of solid-liquid interfacial free energy for molecular systems: TIP4P ice-water interface. Phys. Rev. Lett. 100, 036104 (2008).

62. Espinosa, J. R., Vega, C. \& Sanz, E. Ice-water interfacial free energy for the TIP4P, TIP4P/2005, TIP4P/ice, and mW models as obtained from the mold integration technique. J. Phys. Chem. C 120, 8068-8075 (2016).
63. Vega, C., Sanz, E. \& Abascal, J. L. F. The melting temperature of the most common models of water. J. Chem. Phys. 122, 114507 (2005).

64. Hudait, A., Qiu, S., Lupi, L. \& Molinero, V. Free energy contributions and structural characterization of stacking disordered ices. Phys. Chem. Chem. Phys. 18, 9544-9553 (2016).

\section{Acknowledgements}

The authors thank Maria Chan, Alper Kinaci, Kiran Sasikumar, Al Wagner and Ross Harder for useful discussions. Use of the Center for Nanoscale Materials was supported by the U. S. Department of Energy, Office of Science, Office of Basic Energy Sciences, under Contract No. DE-AC02-06CH11357. This research also used resources of the Argonne Leadership Computing Facility at Argonne National Laboratory, which is supported by the Office of Science of the U.S. Department of Energy under contract DE-AC02-06CH11357. This research used resources of the National Energy Research Scientific Computing Center, a DOE Office of Science User Facility supported by the Office of Science of the U.S. Department of Energy under Contract No. DE-AC0205CH11231. We also acknowledge the Carbon, Fusion and LCRC computing facilities at Argonne.

\section{Author contributions}

H.C., M.J.C., and B.N. contributed equally. H.C., M.J.C., B.N., and S.K.R.S. conceived and designed the project. H.C., B.N., M.J.C., and S.K.R.S. developed the machine learning framework and bond-order potential model for water with input from S.K.G. M.J.C. and H.C. performed the large-scale simulations of grain formation and growth. H.C., B.N., and M.J.C. developed the feature detection algorithm for 3D analysis of grain size and distribution. T.D.L. calculated free energies of various stacked disorder ice phases. B.N performed CI-NEB calculations of the energy barrier for elimination of a stacking fault plane in hexagonal ice. S.K.R.S. supervised the overall project. All the authors including C.J.B. and S.K.G. performed the data analysis and contributed to the preparation of the manuscript.

\section{Additional information}

Supplementary Information accompanies this paper at https://doi.org/10.1038/s41467018-08222-6.

Competing interests: The authors declare no competing interests.

Reprints and permission information is available online at http://npg.nature.com/ reprintsandpermissions/

Journal peer review information: Nature Communications thanks the anonymous reviewers for their contribution to the peer review of this work.

Publisher's note: Springer Nature remains neutral with regard to jurisdictional claims in published maps and institutional affiliations.

Open Access This article is licensed under a Creative Commons Attribution 4.0 International License, which permits use, sharing, adaptation, distribution and reproduction in any medium or format, as long as you give appropriate credit to the original author(s) and the source, provide a link to the Creative Commons license, and indicate if changes were made. The images or other third party material in this article are included in the article's Creative Commons license, unless indicated otherwise in a credit line to the material. If material is not included in the article's Creative Commons license and your intended use is not permitted by statutory regulation or exceeds the permitted use, you will need to obtain permission directly from the copyright holder. To view a copy of this license, visit http://creativecommons.org/ licenses/by/4.0/.

This is a U.S. government work and not under copyright protection in the U.S.; foreign copyright protection may apply 2019 